\title{
Hölder estimates for trajectories of differential inclusions and HJB equations with state constraints
}

\author{
Giancarlo Facchi and Daniel Hoehener
}

\begin{abstract}
We consider the differential inclusion $\dot{x} \in F(t, x)$, with $F$ measurable in $t$ and Lipschitz in $x$, together with the state constraint $x(t) \in \Omega$ and prove the existence of neighboring trajectories lying in the interior of $\Omega$. Using this result, we can characterize the Value Function of the Bolza Problem as the unique lower semicontinuous solution to the corresponding Hamilton-Jacobi-Bellman equation.
\end{abstract}

Mathematics Subject Classification. 34A60, 34H05, 49K15, 49L25, 93C15. Keywords. Differential inclusion, State constraints, Neighboring feasible trajectories, Hamilton-Jacobi-Bellman equation.

\section{Introduction}

In the present paper we consider the differential inclusion

$$
\dot{x} \in F(t, x),
$$

together with the state constraint

$$
x(t) \in \Omega, \text { for all } t \in[0, T] .
$$

A Carathéodory solution of $(D I)$ will be called an $F$-trajectory. By definition, this is an absolutely continuous map from the interval $[0, T]$ into $\mathbb{R}^{n}$, whose time derivative $\dot{x}(t)=\frac{d}{d t} x(t)$ satisfies the differential inclusion $(D I)$ at almost every time $t$. An $F$-trajectory that satisfies (1.1) is feasible.

In particular, we are interested by the following question.

Q: Suppose a feasible F-trajectory $x^{*}$ is given. Is there an $\varepsilon_{0}>0$ such that for all $x_{0} \in B\left(x^{*}(0), \varepsilon_{0}\right) \cap$ Int $\Omega$ there exists an F-trajectory $x:[0, T] \rightarrow$ Int $\Omega$ satisfying $x(0)=x_{0}$ such that

This work was partially supported by the Commission of the European Communities under the 7th Framework Programme Marie Curie Initial Training Network (FP7-PEOPLE-2010ITN), project SADCO, contract number 264735. 


$$
\left\|x-x^{*}\right\|_{\mathbf{L}^{\infty}([0, T])} \leq \omega(\varepsilon),
$$

where $\varepsilon \doteq\left|x_{0}-x^{*}(0)\right|$ and $\omega:[0, \infty] \rightarrow[0, \infty]$ is such that $\lim _{\varepsilon \rightarrow 0} \omega(\varepsilon)=$ $\omega(0)=0$ ?

A related, more general problem is the following.

$\mathbf{Q}^{\prime}$ : Suppose an F-trajectory $x^{*}$ is given. For any point $x_{0} \in \Omega$ we define $\max _{t \in[0, T]} \operatorname{dist}\left(x^{*}(t), \Omega\right)+\left|x^{*}(0)-x_{0}\right| \doteq \varepsilon$. Assuming $\varepsilon>0$ small enough, is there an F-trajectory $x:[0, T] \rightarrow \Omega$ such that $x(0)=x_{0}$ and

$$
\left\|x-x^{*}\right\|_{\mathbf{L}^{\infty}([0, T])} \leq \omega(\varepsilon),
$$

where $\omega(\cdot)$ is as above?

The $F$-trajectory $x$ is generally referred to as a neighboring feasible trajectory (NFT).

In the case where the set $\Omega$ has a smooth boundary, Frankowska and Rampazzo [14] showed that, assuming an inward pointing condition (first introduced by Soner $[16,17])$, one can answer the second question affirmatively under quite general assumptions on $F$, like measurability in time and Lipschitz continuity in $x$, by choosing a linear function $\omega(\cdot)$ in (1.3).

The situation is more complicated however, if the set $\Omega$ is allowed to have corners. In this case, Bressan and Facchi [6] have shown that, if $F$ does not depend on $t$ and is Lipschitz continuous in the space variable $x$, roughly the same conclusions as in [14] are still valid. In [3-5] the case when $F$ depends on $t$ is analyzed. In particular, in [5] it is shown that there exist dynamics $F$, measurable with respect to $t$ and Lipschitz in $x, \Omega \subset \mathbb{R}^{n}$ and an $F$-trajectory $x^{*}$, with $x^{*}(0) \in \Omega$ and $0<\max _{t \in[0, T]} \operatorname{dist}\left(x^{*}(t), \Omega\right) \doteq \varepsilon$ such that for all feasible $F$-trajectories $x$ starting at $x^{*}(0)$, we have

$$
\left\|x^{*}-x\right\|_{\mathbf{L}^{\infty}([0, T])}>K \varepsilon^{\beta},
$$

for any $K>0$ and any $\beta \in] 0,1[$. Thus in the classical NFT setting (question $\mathbf{Q}^{\prime}$ ), it is in general not possible to obtain Hölder estimates if $F$ depends measurably on $t$.

In the first part of the present paper we show that the first question has a positive answer when $F(t, x)$ is Lipschitz in $x$ and just measurable in $t$, under very general hypotheses on the shape of $\Omega$, and that we can choose $\omega(\varepsilon)=K \varepsilon^{\beta}$, in (1.2), for any $\left.\beta \in\right] 0,1\left[\right.$. Hence the assumption that $x^{*}$ is feasible leads to an estimate that does not hold in the more general setting. This is in particular interesting since there are many applications of NFT theorems where $x^{*}$ is feasible.

In the second part of this paper we give an example of such an application. More precisely, we use the NFT result in order to derive some properties of the Value Function for Optimal Control Problems with state constraints.

It is well known (see for instance [18]) that the differential inclusion (DI) arises in relation to control systems of the form

$$
\left\{\begin{array}{l}
\dot{x}(t)=f(t, x(t), u(t)), u(t) \in U(t), \quad \text { a.e. in }[0, T], \\
x(0)=x_{0} .
\end{array}\right.
$$


We will consider the Bolza problem

$$
\text { Minimize } g(x(T))+\int_{0}^{T} L(t, x(t), u(t)) d t,
$$

over solutions of the control system (1.4), satisfying a state constraint of the form (1.1). Here, $g$ is a lower semicontinuous extended real valued map. Endpoint constraints of the form $x(T) \in C$, for some arbitrary closed set $C \subset \mathbb{R}^{n}$, can be incorporated by supposing that $\operatorname{Dom}(g)=C$.

It is known that the Value Function for such a problem might be discontinuous. Characterizations of the Value Function as the unique lower semicontinuous solution of the Hamilton-Jacobi-Bellman equation in the absence of state constraints were provided by Barron and Jensen [2] and Frankowska [9]. In $[11,12,15]$ these results were extended to problems with state constraints. In [11] state constraints given by an arbitrary closed set with nonempty interior were considered and in [12] these results were generalized to degenerate sets. In both papers the multifunction $F(t, x)$ is assumed to be Lipschitz in both $t$ and $x$ and it is assumed that the following outward pointing condition holds, given here, for the sake of simplicity, in the case when $\Omega$ has a smooth boundary and nonempty interior:

$$
\max _{u \in U(t)}\left\langle n_{x}, f(t, x, u)\right\rangle>0, \quad \text { for all } x \in \partial \Omega,
$$

where $n_{x}$ is the outward unit normal to $\Omega$ at $x$. In [15] similar results were deduced for dynamics that are continuous with respect to the time variable but under the assumption that the set $\Omega$ has a smooth boundary. In the present paper we work in a framework where the data is merely measurable in time and we assume that $\Omega$ is a finite intersection of smooth sets. In this setting we show that the Value Function can still be characterized as the unique lower semicontinuous solution of the Hamilton-Jacobi-Bellman equation, in the sense that was introduced in [13] in the absence of state constraints.

The paper is organized as follows. In the second section we will introduce some notations and recall a viability theorem due to Frankowska and Plaskacz (see [10]) for differential inclusions with dynamics that are merely measurable in time and upper semicontinuous in the state variable. In Sect. 3 we state and prove an NFT theorem that provides a positive answer to the question $\mathbf{Q}$ from above. Finally, in Sect. 4 we use the results of the previous sections in order to characterize the Value Function as the unique lower semicontinuous solution of the Hamilton-Jacobi-Bellman equation.

\section{Preliminaries}

\subsection{Basic notations and definitions}

Let $S \subseteq \mathbb{R}^{n}$. Then Int $S, \bar{S}, \partial S, S^{c}$ and $\overline{c o} S$ denote respectively its interior, its closure, its boundary, its complement and its closed convex hull. $B^{n} \doteq\{x \in$ $\left.\mathbb{R}^{n}|| x \mid<1\right\}$ denotes the open unit ball, $S^{n-1}$ stands for the unit sphere, i.e. $\partial B^{n}$ and $B^{n}(x, r)$ is the open ball with radius $r>0$ and center $x \in \mathbb{R}^{n}$. We omit the superscript $n$ if the dimension is clear by the context. 
For a closed subset $K \subseteq \mathbb{R}^{n}$ and $x \in K$, we denote by $T_{K}(x)$ and $C_{K}(x)$ respectively the Bouligand and the Clarke tangent cones of $K$ at $x$. See for instance [1] for their definitions. The set $K$ is called sleek, if these tangent cones coincide for all $x \in K$. In this case the set-valued map $x \rightsquigarrow T_{K}(x)$ is lower semicontinuous at every point of $K$. For further properties of sleek sets, see for instance [1].

Definition. Let $K$ be a closed subset of $\mathbb{R}^{n}$. The (negative) polar cone to $K$ is the set defined by

$$
K^{-} \doteq\left\{x \in \mathbb{R}^{n} \mid\langle x, k\rangle \leq 0, \text { for all } k \in K\right\} .
$$

The normal cone to $K$ at $x$ is the set $N_{K}(x) \doteq C_{K}(x)^{-}$.

Let $\mathcal{T}$ be a metric space and $\left\{A_{\tau}\right\}_{\tau \in \mathcal{T}}$ be a family of subsets of a metric space $X$. The upper and the lower limit of $A_{\tau}$ at $\tau_{0} \in \mathcal{T}$ are defined as follows:

$$
\begin{aligned}
& \underset{\tau \rightarrow \tau_{0}}{\operatorname{Limsup}} A_{\tau}=\left\{v \in X \mid \liminf _{\tau \rightarrow \tau_{0}} \operatorname{dist}\left(v, A_{\tau}\right)=0\right\}, \\
& \operatorname{Liminf}_{\tau \rightarrow \tau_{0}} A_{\tau}=\left\{v \in X \mid \lim _{\tau \rightarrow \tau_{0}} \operatorname{dist}\left(v, A_{\tau}\right)=0\right\} .
\end{aligned}
$$

The set $A$ is the limit of $A_{\tau}$ if $A=\operatorname{Liminf}_{\tau \rightarrow \tau_{0}} A_{\tau}=\operatorname{Limsup}_{\tau \rightarrow \tau_{0}} A_{\tau} \doteq$ $\operatorname{Lim}_{\tau \rightarrow \tau_{0}} A_{\tau}$.

Finally, we are going to introduce the basic notions of nonsmooth analysis that we will use in the rest of the paper.

In the following $\varphi: \mathbb{R}^{n} \rightarrow \mathbb{R} \cup\{+\infty\}$ is an extended function and its domain, denoted by $\operatorname{Dom}(\varphi)$, is the set of all $x \in \mathbb{R}^{n}$ such that $\varphi(x)<+\infty$. The graph of $\varphi$ is denoted by $\operatorname{Gr} \varphi$ and its epigraph, i.e. the set $\{(x, y) \mid \varphi(x) \leq y\}$, by $\mathcal{E} p \varphi$.

Definition. The subdifferential of $\varphi$ at $x_{0} \in \operatorname{Dom}(\varphi)$ is given by

$$
\partial_{-} \varphi\left(x_{0}\right) \doteq\left\{p \in \mathbb{R}^{n} \mid \liminf _{x \rightarrow x_{0}} \frac{\varphi(x)-\varphi\left(x_{0}\right)-\left\langle p, x-x_{0}\right\rangle}{\left|x-x_{0}\right|} \geq 0\right\} .
$$

Definition. The contingent epiderivative of $\varphi$ at $x_{0} \in \operatorname{Dom}(\varphi)$ in the direction $u \in \mathbb{R}^{n}$ is defined by

$$
D_{\uparrow} \varphi\left(x_{0}\right)(u) \doteq \liminf _{\substack{h \rightarrow 0+\\ u^{\prime} \rightarrow u}} \frac{\varphi\left(x_{0}+h u^{\prime}\right)-\varphi\left(x_{0}\right)}{h} .
$$

Note that the contingent epiderivative can be characterized by the following identity (see [1, pp. 226-229] for a proof):

$$
\mathcal{E} p D_{\uparrow} \varphi\left(x_{0}\right)=T_{\mathcal{E} p \varphi}\left(x_{0}, \varphi\left(x_{0}\right)\right) .
$$

The subdifferential and the contingent epiderivative are linked by the following proposition from [8, Lemma 2.7] (see also [1, pp. 249, 253]).

Proposition 2.1. Let $x_{0} \in \operatorname{Dom}(\varphi)$ then

$$
\begin{aligned}
p \in \partial_{-} \varphi\left(x_{0}\right) & \Longleftrightarrow \text { for all } u \in \mathbb{R}^{n}, \quad\langle p, u\rangle \leq D_{\uparrow} \varphi\left(x_{0}\right)(u) \\
& \Longleftrightarrow(p,-1) \in\left[T_{\mathcal{E}_{p} \varphi}\left(x_{0}, \varphi\left(x_{0}\right)\right)\right]^{-} .
\end{aligned}
$$




\subsection{Some elements of viability theory}

We begin this section with the notion of an absolutely continuous set-valued map, which is defined analogously to the single-valued case.

Definition. The set-valued map $P:[0, T] \rightsquigarrow \mathbb{R}^{n}$ is absolutely continuous if for every $\varepsilon>0$ and every compact set $K$ there exists $\delta>0$, such that whenever a finite sequence of pairwise disjoint subintervals $\left\{\left[t_{i}, \tau_{i}\right]\right\}_{i}$ satisfies, $\sum_{i}\left|\tau_{i}-t_{i}\right| \leq$ $\delta$, then

$$
\sum_{i} \max \left\{e\left(P\left(t_{i}\right) \cap K, P\left(\tau_{i}\right)\right), e\left(P\left(\tau_{i}\right) \cap K, P\left(t_{i}\right)\right\} \leq \varepsilon,\right.
$$

where $e(U, V) \doteq \inf \{\rho>0 \mid U \subset V+\rho B\}$, i.e. $e(U, V)$ is the asymmetric Hausdorff distance from $U$ to $V$.

Remark 2.1. Note that if $P:[0, T] \rightsquigarrow \mathbb{R}^{n}$ is an absolutely continuous setvalued map and $S \subset \mathbb{R}^{n}$ is closed, then the set-valued maps $\widetilde{P}, \widehat{P}:[0, T] \rightsquigarrow \mathbb{R}^{n}$, defined by $\widetilde{P}(t)=P(T-t)$ and $\widehat{P}(t)=P(t) \cup S$ respectively, are absolutely continuous.

Next, let $F:[0, T] \times \mathbb{R}^{n} \rightsquigarrow \mathbb{R}^{n}$ be a set-valued map, $P$ as above, $x_{0} \in \mathbb{R}^{n}$ and consider the differential inclusion:

$$
\left\{\begin{array}{l}
\dot{x}(t) \in F(t, x(t)) \quad \text { a.e. in }\left[t_{0}, T\right] \\
x\left(t_{0}\right)=x_{0}
\end{array}\right.
$$

with the state constraint

$$
x(t) \in P(t) \text { for all }\left[t_{0}, T\right] .
$$

The following theorem is due to Frankowska and Plaskacz [10] (see also [13]).

Theorem 2.2 (Viability Theorem). Assume that a closed valued map $P:[0, T]$ $\rightsquigarrow \mathbb{R}^{n}$ is absolutely continuous, that $F:[0, T] \times \mathbb{R}^{n} \rightsquigarrow \mathbb{R}^{n}$ has closed convex values and

(a) $x \rightsquigarrow F(t, x)$ is upper semicontinuous for almost all $t \in[0, T]$;

(b) $F(\cdot, \cdot)$ is Lebesgue-Borel measurable;

(c) There exists $\mu \in L^{1}\left((0, T) ; \mathbb{R}_{+}\right)$such that

$$
\sup _{v \in F(t, x)}|v| \leq \mu(t)(1+|x|) \quad \text { for a.e. } t \in[0, T] \text { and all } x \in \mathbb{R}^{n} .
$$

Then the following statements are equivalent:

(i) For almost all $t_{0} \in[0, T]$ and $x_{0} \in P\left(t_{0}\right)$,

$$
\left(\{1\} \times F\left(t_{0}, x_{0}\right)\right) \cap \overline{c o} T_{\mathrm{Gr} P}\left(t_{0}, x_{0}\right) \neq \emptyset ;
$$

(ii) For all $t_{0} \in\left[0, T\left[\right.\right.$ and $x_{0} \in P\left(t_{0}\right)$ there exists a solution $x(\cdot)$ of $(2.2)$ satisfying (2.3). 


\section{A neighboring feasible trajectories theorem}

In this section we consider the differential inclusion $(D I)$, together with the time independent state constraint (1.1).

Suppose that $x^{*}(\cdot)$ is a feasible $F$-trajectory. We want to show that under some conditions there exists $\varepsilon_{0}>0$ such that, for all $x_{0} \in B\left(x^{*}(0), \varepsilon_{0}\right) \cap \operatorname{Int} \Omega$, it is possible to construct a second $F$-trajectory $x(\cdot)$, starting at $x_{0}$, whose values are contained in the interior of $\Omega$ and such that

$$
\left\|x-x^{*}\right\|_{\mathbf{L}^{\infty}}=\mathcal{O}\left(\left|x_{0}-x^{*}(0)\right|^{\beta}\right),
$$

where $\beta \in] 0,1[$.

We assume that the set $\Omega \subset \mathbb{R}^{n}$ satisfies the following assumption.

(SC) $\Omega$ is compact and has the representation

$$
\Omega \doteq\left\{x \in \mathbb{R}^{n} \mid \phi_{i}(x) \geq 0, \text { for all } i=1, \ldots, N\right\},
$$

where $\phi_{i} \in \mathcal{C}_{\text {loc }}^{1,1}\left(\mathbb{R}^{n} ; \mathbb{R}\right)$, and at every point $x \in \mathbb{R}^{n}$, if $\phi_{i_{1}}(x)=\cdots=$ $\phi_{i_{\nu}}(x)=0$, then the gradients $\nabla \phi_{i_{1}}(x), \ldots, \nabla \phi_{i_{\nu}}(x)$ are linearly independent.

We also make the following assumptions on the multifunction $F$.

(A1) $F(t, x)$ is closed and nonempty, for every $(t, x) \in[0, T] \times \mathbb{R}^{n}$;

(A2) $t \rightsquigarrow F(t, x)$ is measurable for every $x \in \mathbb{R}^{n}$;

(A3) $x \rightsquigarrow F(t, x)$ is Lipschitz continuous, with Lipschitz constant $L$ for almost every $t \in[0, T]$

(A4) There exists $M>0$ such that

$$
\sup _{v \in F(t, x)}|v| \leq M, \text { for all }(t, x) \in[0, T] \times \mathbb{R}^{n} .
$$

Finally, we will need the following inward pointing condition.

$$
\text { (A5) } \begin{aligned}
& \exists \bar{\rho}>0 \text {, such that } \inf _{v \in F(t, x)} \sup _{n \in N_{\Omega}(x) \cap S^{n-1}}\langle n, v\rangle \leq-\bar{\rho} \text {, for all }(t, x) \in \\
& {[0, T] \times \Omega \text {. }}
\end{aligned}
$$

The main result is as follows.

Theorem 3.1. Assume (SC) and (A1)-(A5). Then there exists $K>0$ such that, for any $\beta \in] 0,1\left[\right.$, given any $F$-trajectory $x^{*}:[0, T] \rightarrow \Omega$, we can find $\varepsilon_{0} \doteq \varepsilon_{0}\left(x^{*}(0)\right)>0$ such that, if $x_{0} \in$ Int $\Omega \cap B\left(x^{*}(0), \varepsilon_{0}\right)$, it is possible to construct a second F-trajectory $x:[0, T] \rightarrow$ Int $\Omega$, with $x(0)=x_{0}$, satisfying

$$
\left\|x-x^{*}\right\|_{\mathbf{L}^{\infty}([0, T])} \leq K \varepsilon^{\beta},
$$

where

(i) if $x_{0} \neq x^{*}(0), \varepsilon=\left|x_{0}-x^{*}(0)\right|<\varepsilon_{0}$;

(ii) if $x_{0}=x^{*}(0)$, $\varepsilon$ is any positive number smaller than $\varepsilon_{0}$.

Remark 3.1. Note that in the above theorem $\varepsilon_{0}$ depends on $x^{*}(0)$ and on the reference trajectory $x^{*}(\cdot)$.

The proof will be achieved by first showing that the conclusions of the above Theorem hold when the state constraint set is

$$
\Gamma \doteq \mathbb{R}_{+}^{m} \times \mathbb{R}^{n-m}
$$


The generalization to a set $\Omega$ of the form (SC) is obtained by a change of coordinates and a covering argument.

We start by proving an easy property of the set $\Gamma$.

Lemma 3.2. Let $\Gamma$ be as in (3.3). Then, for any $z \in \Gamma$, there exists $\kappa(z)>0$ such that

$$
x \in B(z, \kappa(z)) \cap(\Gamma \backslash\{z\}) \Longrightarrow x+[0, \kappa(z)] \frac{x-z}{|x-z|} \subset \Gamma .
$$

Proof. The set $\Gamma$ can be represented as

$$
\Gamma \doteq\left\{x \in \mathbb{R}^{n} \mid\left\langle A x, \mathbf{e}_{i}\right\rangle \leq 0, \text { for all } i \in\{1, \ldots, m\}\right\},
$$

where $A=\left[\begin{array}{cc}-I_{m} & 0 \\ 0 & 0\end{array}\right] \in \mathbb{R}^{n \times n}$, and $I_{m}$ is the $m \times m$ identity matrix. For $z \in \Gamma$, denote by $\mathcal{I}(z) \subseteq\{1, \ldots, m\}$ the set of active indices, that is

$$
i \in \mathcal{I}(z) \Longleftrightarrow\left\langle A z, \mathbf{e}_{i}\right\rangle=0,
$$

where $\mathbf{e}_{i}, i=\{1, \ldots, n\}$, are the canonical basis vectors of $\mathbb{R}^{n}$. In addition, let $\mathcal{I}^{c}(z) \doteq\{1, \ldots, m\} \backslash \mathcal{I}(z)$ denote its complement. We notice that $\mathcal{I}(\cdot)$ is upper semicontinuous, in the sense that for any $z \in \Gamma$ there exists $\delta(z)>0$ such that

$$
\left\{\begin{array}{l}
x \in B(z, \delta(z)) \cap \Gamma \Longrightarrow \mathcal{I}(x) \subseteq \mathcal{I}(z) \\
x \in B(z, \delta(z)) \text { and } j \in \mathcal{I}^{c}(z) \Longrightarrow\left\langle A x, \mathbf{e}_{j}\right\rangle<0 .
\end{array}\right.
$$

Let $z \in \Gamma$. We claim that $\kappa(z) \doteq \delta(z) / 2$, where $\delta(z)$ is as in (3.6) satisfies (3.4). Indeed, let $x \in B(z, \kappa(z)) \cap(\Gamma \backslash\{z\})$, and $y \doteq x+\kappa \frac{x-z}{|x-z|}$, for some $\kappa \in[0, \kappa(z)]$. Let $i^{\prime} \in \mathcal{I}(z)$ and let $j^{\prime} \in \mathcal{I}^{c}(z)$. Then, by using (3.5) and (3.6), we obtain

$$
\left\langle A y, \mathbf{e}_{i^{\prime}}\right\rangle=\left\langle A x, \mathbf{e}_{i^{\prime}}\right\rangle+\frac{\kappa}{|x-z|}\left\langle A(x-z), \mathbf{e}_{i^{\prime}}\right\rangle=\left(1+\frac{\kappa}{|x-z|}\right)\left\langle A x, \mathbf{e}_{i^{\prime}}\right\rangle \leq 0
$$

and also, since $y \in B(x, \kappa(z)) \subset B(z, \delta(z))$, we see that

$$
\left\langle A y, \mathbf{e}_{j^{\prime}}\right\rangle<0 \text {. }
$$

Hence $y \in \Gamma$.

The following Local Lemma proves Theorem 3.1 in the special case when the state constraint set is the cone $\Gamma$, and provided that $T$ is sufficiently small.

Lemma 3.3. Let $\Gamma$ be as in (3.3), $\left(t_{0}, z\right) \in[0, T] \times \Gamma$ and $\tilde{r}>0$. Further assume that $F:[0, T] \times B(z, \tilde{r}) \rightsquigarrow \mathbb{R}^{n}$ satisfies $(\mathbf{A 1})-(\mathbf{A 4})$ on $[0, T] \times B(z, \tilde{r})$ and $\exists \bar{\delta}>0, \bar{\rho}>0$, such that $\inf _{v \in F(t, z)} \sup _{n \in N_{\Gamma}(z) \cap S^{n-1}}\langle n, v\rangle \leq-4 \bar{\rho}$, for all $t \in\left[t_{0}, t_{0}+\bar{\delta}\right]$.

Then there exist positive constants $r_{z}^{*}, T_{z}^{*}, K_{z}^{*}$, such that, given any feasible F-trajectory $x^{*}(\cdot)$ with

$$
\left|x^{*}\left(t_{0}\right)-z\right| \leq r_{z}^{*},
$$

and any $\gamma \in] 0,1\left[\right.$, there exists $\varepsilon_{z}^{*}>0$ such that for all $x_{0} \in B\left(x^{*}\left(t_{0}\right), \varepsilon_{z}^{*}\right) \cap \operatorname{Int} \Gamma$ the following holds: 
(i) If $x^{*}\left(t_{0}\right) \neq x_{0}$, then there exists an $F$-trajectory $x:\left[t_{0}, t_{0}+T_{z}^{*}\right] \rightarrow \operatorname{Int} \Gamma$, such that $x\left(t_{0}\right)=x_{0}$, and

$$
\left\|x-x^{*}\right\|_{\mathbf{L} \infty\left(\left[t_{0}, t_{0}+T_{z}^{*}\right]\right)} \leq K_{z}^{*}\left|x_{0}-x^{*}\left(t_{0}\right)\right|^{\gamma} ;
$$

(ii) If $x^{*}\left(t_{0}\right)=x_{0}$, then for any $\left.\varepsilon^{\prime} \in\right] 0,1[$, there exists an $F$-trajectory $x:\left[t_{0}, t_{0}+T_{z}^{*}\right] \rightarrow$ Int $\Gamma$, such that $x\left(t_{0}\right)=x_{0}$, and

$$
\left\|x-x^{*}\right\|_{\mathbf{L}^{\infty}\left(\left[t_{0}, t_{0}+T_{z}^{*}\right]\right)} \leq K_{z}^{*} \varepsilon^{\prime} .
$$

Proof. Let us first assume that $F(t, x)$ is convex for all $(t, x) \in[0, T] \times B(z, \tilde{r})$.

1. Since the set $\Gamma$ is sleek and has nonempty interior, $N_{\Gamma}(\cdot) \cap S^{n-1}$ is upper semicontinuous and therefore there exists $\bar{\eta}>0$ such that

$$
\text { for all } x \in B(z, \bar{\eta}), N_{\Gamma}(x) \cap S^{n-1} \subseteq N_{\Gamma}(z) \cap S^{n-1}+\bar{\rho} B .
$$

Consider

$$
0<r_{z}^{*} \leq \min \left\{\frac{\bar{\rho}}{2 L}, \frac{\bar{\eta}}{2}, \frac{\tilde{r}}{4}\right\},
$$

and assume that $\varepsilon_{z}^{*} \leq r_{z}^{*}$ [see (3.14) below]. Then $\left|x_{0}-z\right| \leq 2 r_{z}^{*}$ and by Lipschitz continuity of $F(t, \cdot)$ we get

$$
\inf _{v \in F\left(t, x_{0}\right)} \sup _{n \in N_{\Gamma}(z) \cap S^{n-1}}\langle n, v\rangle \leq-2 \bar{\rho}, \quad \text { for all } t \in\left[t_{0}, t_{0}+\bar{\delta}\right] .
$$

Consequently, there exists a measurable selection $\alpha(s) \in F\left(s, x_{0}\right)$ such that

$$
\langle n, \alpha(s)\rangle \leq-2 \bar{\rho}, \text { for all } n \in N_{\Gamma}(z) \cap S^{n-1} \text {, for all } s \in\left[t_{0}, t_{0}+\bar{\delta}\right] .
$$

For any $t \in\left[t_{0}, t_{0}+\bar{\delta}\right]$, define

$$
v(t) \doteq \int_{t_{0}}^{t} \alpha(s) d s,
$$

and the compact set

$$
V \doteq \overline{\left\{\frac{v(t)}{t-t_{0}} \mid t \in\right] t_{0}, t_{0}+\bar{\delta}[\}} .
$$

Using a covering argument and the properties of Clarke's tangent cone, we can conclude that

$$
\begin{gathered}
\left.\exists \rho_{z} \in\right] 0,1\left[\text { s.t. for all } v \in V, \text { for all } z^{\prime} \in B\left(z, \rho_{z}\right) \cap \Gamma,\right. \\
z^{\prime}+\left[0, \rho_{z}\right] B\left(v, \rho_{z}\right) \subset \Gamma .
\end{gathered}
$$

2. Without loss of generality we assume that $r_{z}^{*} \leq \frac{\rho_{z}}{4}, L \geq 1$ and $M \geq 1$. In addition we define the following constants:

$$
\begin{array}{r}
T_{z}^{*} \doteq \min \left\{1, \bar{\delta}, \frac{\rho_{z}}{12 L M e^{L}}, \frac{\tilde{r}}{2 M}\right\}, \\
K_{z}^{*} \doteq 2 M+2 \geq \frac{1}{2} \rho_{z} T_{z}^{*}+2 M T_{z}^{*}+1 .
\end{array}
$$

Fix $\gamma \in] 0,1\left[\right.$ and let the feasible $F$-trajectory $x^{*}(\cdot)$ be given. By Lemma 3.2, there exists $\left.\kappa_{0} \in\right] 0, \frac{\rho_{z}}{2}[$,

$$
\kappa_{0} \leq \kappa\left(x^{*}\left(t_{0}\right)\right)
$$


such that

$$
x \in B\left(x^{*}\left(t_{0}\right), \kappa_{0}\right) \cap\left(\Gamma \backslash\left\{x^{*}\left(t_{0}\right)\right\}\right) \Longrightarrow x+\left[0, \kappa_{0}\right] \frac{x-x^{*}\left(t_{0}\right)}{\left|x-x^{*}\left(t_{0}\right)\right|} \subset \Gamma .
$$

Consider the function $f(y)=y^{1-\gamma}-y$. By direct computation, we see that $f(\cdot)$ is strictly monotone increasing on $\left[0,(1-\gamma)^{\frac{1}{\gamma}}\right]$, hence there exists $\left.\bar{y} \in] 0,(1-\gamma)^{\frac{1}{\gamma}}\right]$ with the property that, for every $y \leq \bar{y}, f(y) \leq \kappa_{0}$. Let

$$
\varepsilon_{z}^{*} \doteq \min \left\{r_{z}^{*}, \kappa_{0}, \bar{y},\left(\frac{\rho_{z}}{4 L e^{L}}\right)^{\frac{1}{1-\gamma}}, \frac{\tilde{r}}{8}, 8^{-\frac{1}{\gamma}}\right\} .
$$

3. Consider the case when $x_{0} \neq x^{*}\left(t_{0}\right)$ and set $\varepsilon \doteq\left|x_{0}-x^{*}\left(t_{0}\right)\right|$. We assume that

$$
\varepsilon<\varepsilon_{z}^{*}
$$

Then, define $w \in \mathbb{R}^{n}$ as

$$
w \doteq x^{*}\left(t_{0}\right)+\varepsilon^{-\gamma}\left(x_{0}-x^{*}\left(t_{0}\right)\right) \text {. }
$$

This implies

$$
\left|w-x^{*}\left(t_{0}\right)\right|=\varepsilon^{-\gamma}\left|x_{0}-x^{*}\left(t_{0}\right)\right|=\varepsilon^{1-\gamma},
$$

hence (since $\varepsilon<\varepsilon_{z}^{*} \leq \bar{y}$ ),

$$
\left|w-x_{0}\right|=\left|x^{*}\left(t_{0}\right)-x_{0}+\varepsilon^{-\gamma}\left(x_{0}-x^{*}\left(t_{0}\right)\right)\right|=\varepsilon^{1-\gamma}-\varepsilon \leq \kappa_{0} .
$$

Therefore $w \in \Gamma$ by (3.13). Define

$$
y(t) \doteq w+v(t), \quad \text { for all } t \in\left[t_{0}, t_{0}+T_{z}^{*}\right] .
$$

Then, since $w \in B\left(x_{0}, \kappa_{0}\right) \subseteq B\left(z, \rho_{z}\right)$, using (3.11) we get

$$
\begin{array}{r}
y(t)+\left(t-t_{0}\right) \rho_{z} B=w+v(t)+\left(t-t_{0}\right) \rho_{z} B \\
=w+\left(t-t_{0}\right) B\left(\frac{v(t)}{t-t_{0}}, \rho_{z}\right) \subset \Gamma,
\end{array}
$$

after observing that $T_{z}^{*} \leq \rho_{z}$ by definition.

Finally, define

$$
y_{\varepsilon}(t) \doteq \varepsilon^{\gamma} y(t)+\left(1-\varepsilon^{\gamma}\right) x^{*}(t), \quad t \in\left[t_{0}, t_{0}+T_{z}^{*}\right] .
$$

Clearly we have

$$
y_{\varepsilon}\left(t_{0}\right)=\varepsilon^{\gamma} w+\left(1-\varepsilon^{\gamma}\right) x^{*}\left(t_{0}\right)=x_{0},
$$

and

$$
y_{\varepsilon}(t)+\left(t-t_{0}\right) \varepsilon^{\gamma} \rho_{z} B=\varepsilon^{\gamma}\left(y(t)+\left(t-t_{0}\right) \rho_{z} B\right)+\left(1-\varepsilon^{\gamma}\right) x^{*}(t) \subset \Gamma,
$$

as a consequence of (3.19) and the convexity of $\Gamma$.

4. We want to prove the existence of a feasible $F$-trajectory by applying Filippov's theorem (see [7]). First notice that

$$
\begin{aligned}
\left|x^{*}(t)-y_{\varepsilon}(t)\right| & =\varepsilon^{\gamma}\left|x^{*}(t)-y(t)\right| \leq \varepsilon^{\gamma}\left(\left|y(t)-y\left(t_{0}\right)\right|+\left|x^{*}(t)-x^{*}\left(t_{0}\right)\right|+\left|w-x^{*}\left(t_{0}\right)\right|\right) \\
& \leq \varepsilon^{\gamma}\left(2 M\left(t-t_{0}\right)+\varepsilon^{1-\gamma}\right),
\end{aligned}
$$


where the last inequality follows from (3.17). Furthermore, by (3.10), (3.12), (3.14) and (3.15), we find that for all $t \in\left[t_{0}, t_{0}+T_{z}^{*}\right]$,

$$
\left|z-y_{\varepsilon}(t)\right| \leq\left|z-x^{*}(t)\right|+\left|x^{*}(t)-y_{\varepsilon}(t)\right| \leq \frac{3 \tilde{r}}{4}+\varepsilon^{\gamma} \tilde{r}+\varepsilon<\tilde{r} .
$$

Hence $y_{\varepsilon}\left(\left[t_{0}, t_{0}+T_{z}^{*}\right]\right) \subset B(z, \tilde{r})$. Next we estimate the distance between

$$
\dot{y}_{\varepsilon}(t)=\varepsilon^{\gamma} \dot{y}(t)+\left(1-\varepsilon^{\gamma}\right) \dot{x}^{*}(t)
$$

and the admissible velocity set $F\left(t, y_{\varepsilon}(t)\right)$. We have that,

$$
\begin{aligned}
\dot{y}(t)=\alpha(t) \in F\left(t, x_{0}\right) & \subseteq F\left(t, y_{\varepsilon}(t)\right)+L\left|x_{0}-y_{\varepsilon}(t)\right| B \\
& \subseteq F\left(t, y_{\varepsilon}(t)\right)+L M\left(t-t_{0}\right) B .
\end{aligned}
$$

Next, observe that,

$$
\begin{aligned}
\dot{x}^{*}(t) \in F\left(t, x^{*}(t)\right) & \subset F\left(t, y_{\varepsilon}(t)\right)+L\left|x^{*}(t)-y_{\varepsilon}(t)\right| B \\
& \subset F\left(t, y_{\varepsilon}(t)\right)+L\left(2 M\left(t-t_{0}\right) \varepsilon^{\gamma}+\varepsilon\right) B .
\end{aligned}
$$

From (3.23), (3.24) and (3.25), we see that

$$
\dot{y}_{\varepsilon}(t) \in F\left(t, y_{\varepsilon}(t)\right)+L\left(3 M\left(t-t_{0}\right) \varepsilon^{\gamma}+\varepsilon\right) B .
$$

By Filippov's theorem, we conclude that there exists an $F$-trajectory $x(\cdot)$ such that

$$
\begin{aligned}
\left|x(t)-y_{\varepsilon}(t)\right| & \leq e^{L\left(t-t_{0}\right)} \int_{t_{0}}^{t} L\left(3 M\left(s-t_{0}\right) \varepsilon^{\gamma}+\varepsilon\right) d s \\
& \leq e^{L T_{z}^{*}} L\left(3 M T_{z}^{*} \varepsilon^{\gamma}+\varepsilon\right)\left(t-t_{0}\right) \\
& \leq\left[3 L M T_{z}^{*} e^{L T_{z}^{*}} \varepsilon^{\gamma}+L e^{L T_{z}^{*}} \varepsilon\right]\left(t-t_{0}\right) \leq \frac{\rho_{z}}{2} \varepsilon^{\gamma}\left(t-t_{0}\right),
\end{aligned}
$$

where the last inequality follows from the definitions (3.12) and (3.14) of $T_{z}^{*}$ and $\varepsilon_{z}^{*}$. We also observe that (3.21) and (3.26) together imply that $x\left(\left[t_{0}, t_{0}+\right.\right.$ $\left.\left.T_{z}^{*}\right]\right) \subset$ Int $\Gamma$. follows:

5. The $\mathbf{L}^{\infty}$ estimate (3.8) can be computed by using (3.22) and (3.26) as

$$
\begin{aligned}
\left|x(t)-x^{*}(t)\right| & \leq\left|x(t)-y_{\varepsilon}(t)\right|+\left|y_{\varepsilon}(t)-x^{*}(t)\right| \\
& \leq \frac{\rho_{z}}{2} \varepsilon^{\gamma} T_{z}^{*}+\varepsilon^{\gamma}\left(2 M T_{z}^{*}+\varepsilon^{1-\gamma}\right) \\
& \leq K_{z}^{*} \varepsilon^{\gamma},
\end{aligned}
$$

where $K_{z}^{*}$ is given by (3.12).

6. If $x^{*}\left(t_{0}\right)=x_{0}$ we notice that we do not need to define $w$ as in (3.16) and simply define

$$
y(t) \doteq x_{0}+v(t) .
$$

Take any $\left.\varepsilon^{\prime} \in\right] 0,1[$ and as in $(3.20)$ we can define,

$$
y_{\varepsilon^{\prime}}(t) \doteq \varepsilon^{\prime} y(t)+\left(1-\varepsilon^{\prime}\right) x^{*}(t) .
$$


By the same argument we obtain (3.9). Indeed, we get $\dot{y}_{\varepsilon^{\prime}}(t) \in F\left(t, y_{\varepsilon^{\prime}}(t)\right)+\left(3 \varepsilon^{\prime}-\varepsilon^{\prime 2}\right) L M\left(t-t_{0}\right) B \subset F\left(t, y_{\varepsilon^{\prime}}(t)\right)+3 \varepsilon^{\prime} L M\left(t-t_{0}\right) B$, hence

$$
\left|x(t)-y_{\varepsilon^{\prime}}(t)\right| \leq 3 \varepsilon^{\prime} e^{L T_{z}^{*}} L M T_{z}^{*}\left(t-t_{0}\right) \leq \frac{\rho_{z}}{4} \varepsilon^{\prime}\left(t-t_{0}\right)
$$

gives the feasibility of $x(\cdot)$, and we get

$$
\left|x(t)-x^{*}(t)\right| \leq\left|x(t)-y_{\varepsilon^{\prime}}(t)\right|+\left|y_{\varepsilon^{\prime}}(t)-x^{*}(t)\right| \leq K_{z}^{*} \varepsilon^{\prime} .
$$

7. If the values of the multifunction $F$ are not convex, call $\tilde{x}$ the $c o F$ trajectory constructed in the previous steps. Then since $\tilde{x}(t) \in \operatorname{Int} \Gamma$, by the Filippov-Ważewski Theorem (cf. [14]), we can construct $x(\cdot)$ satisfying all the requirements.

Proof of Theorem 3.1. 1. Fix $\beta \in] 0,1[$. By (SC), for every $z \in \Omega$ there exists an open set $V_{z} \subseteq \mathbb{R}^{n}, z \in V_{z}$ such that

$$
\Omega \cap V_{z}=\left\{x \mid \phi_{i}(x) \geq 0, \text { for all } i \in \mathcal{I}(z)\right\} \cap V_{z},
$$

where $\mathcal{I}(z) \subseteq\{1, \ldots, N\}$ has cardinality $m(z) \doteq|\mathcal{I}(z)| \leq n$. Indeed, we have $\mathcal{I}(z)=\left\{i \in\{1, \ldots, N\} \mid \phi_{i}(z)=0\right\}$. Therefore, since by (SC) the vectors $\nabla \phi_{i}(z), i \in \mathcal{I}(z)$ are linearly independent, we have that $m(z) \leq n$. Normalizing if necessary, we can assume that $\left|\nabla \phi_{i}(z)\right|=1$ for all $i \in \mathcal{I}(z)$.

For every $z \in \Omega$, choose $n-m(z)$ unit vectors $\tilde{\mathbf{e}}_{1}^{z}, \ldots, \tilde{\mathbf{e}}_{n-m(z)}^{z}$ forming an orthonormal basis of

$$
\left(\operatorname{span}\left\{\nabla \phi_{i}(z) \mid i \in \mathcal{I}(z)\right\}\right)^{\perp}
$$

and define the map $\Phi^{(z)}: V_{z} \rightarrow \mathbb{R}^{n}$ as follows:

$$
x \mapsto\left(\begin{array}{l}
\phi_{k_{1}}(x) \\
\vdots \\
\phi_{k_{m(z)}}(x) \\
\left\langle x, \tilde{\mathbf{e}}_{1}^{z}\right\rangle \\
\vdots \\
\left\langle x, \tilde{\mathbf{e}}_{n-m(z)}^{z}\right\rangle
\end{array}\right) \doteq \Phi^{(z)}(x)
$$

where

$$
\left\{k_{1}, \ldots, k_{m(z)}\right\}=\mathcal{I}(z) \subseteq\{1, \ldots, N\},
$$

with the understanding that $\Phi^{(z)}(\cdot)$ is the identity map on $\mathbb{R}^{n}$, whenever $\mathcal{I}(z)=\emptyset$. By the Inverse Function Theorem, there exist $\tilde{r}_{1}(z), \tilde{r}_{2}(z)>0$ such that $\Phi^{(z)}$ is a $\mathcal{C}^{1,1}$ diffeomorphism from an open set $\widetilde{V}_{z} \supset B\left(z, \tilde{r}_{1}(z)\right)$ into the open ball $B\left(\Phi^{(z)}(z), \tilde{r}_{2}(z)\right)$. In addition, we note that $\Phi^{(z)}\left(V_{z} \cap \Omega\right) \subset$ $\mathbb{R}_{+}^{m(z)} \times \mathbb{R}^{n-m(z)} \doteq \Gamma^{(z)}$.

2. Define the set-valued map $\widetilde{F}^{(z)}:[0, T] \times B\left(\Phi^{(z)}(z), \tilde{r}_{2}(z)\right) \rightarrow \mathbb{R}^{n}$ by

$$
\widetilde{F}^{(z)}(t, \xi) \doteq D \Phi^{(z)}\left(\left(\Phi^{(z)}\right)^{-1}(\xi)\right) \cdot F\left(t,\left(\Phi^{(z)}\right)^{-1}(\xi)\right) \text {. }
$$


Here $D \Phi^{(z)}$ denotes the differential of the map $x \mapsto \Phi^{(z)}(x)$. Notice that the multifunction $\widetilde{F}^{(z)}$ satisfies the assumptions (A1)-(A4). Let us show that (3.7) holds for $\bar{\xi} \doteq \Phi^{(z)}(z)$. Fix $t_{0} \in[0, T$ [ arbitrary. If $z \in$ Int $\Omega$ then $m(z)=0$, thus $\Gamma^{(z)}=\mathbb{R}^{n}$ and (3.7) is trivially satisfied. On the other hand, if $z \in \partial \Omega$, then $\bar{\xi} \in \partial \Gamma^{(z)}$ and we have that

$$
\begin{aligned}
& N_{\Gamma^{(z)}}(\bar{\xi}) \cap S^{n-1} \\
& \quad=\left\{\left(n_{1}, \ldots, n_{n}\right) \in S^{n-1} \mid n_{i} \leq 0 \text { if } i \in\{1, \ldots, m(z)\} \text { and } n_{i}=0 \text { otherwise }\right\} .
\end{aligned}
$$

Furthermore, by (3.27) the normal cone to $\Omega$ at $z$ is given by

$$
N_{\Omega}(z)=\overline{c o}\left\{-\nabla \phi_{i}(z) \mid i \in \mathcal{I}(z)\right\} .
$$

Finally, by (A5) there exists $\bar{\rho}>0$ such that for all $t \in\left[t_{0}, T\right]$ and all $n \in$ $N_{\Omega}(z) \cap S^{n-1}$, there exists $v \in F(t, z)$ such that

$$
\langle n, v\rangle \leq-\bar{\rho} .
$$

Fix an arbitrary $t \in\left[t_{0}, T\right]$ and let $v \in F(t, z)$ be such that (3.28) is satisfied. Then, since

$$
D \Phi^{(z)}(z)=\left(\begin{array}{l}
D \phi_{k_{1}}(z) \\
\vdots \\
D \phi_{k_{m(z)}}(z) \\
\left(\mathbf{e}_{1}^{z}\right)^{T} \\
\vdots \\
\left(\mathbf{e}_{n-m(z)}^{z}\right)^{T}
\end{array}\right)
$$

we find that

$$
\widetilde{F}^{(z)}(t, \bar{\xi}) \ni D \Phi^{(z)}(z) v \doteq \tilde{v}
$$

where $\tilde{v}^{T}=\left(\tilde{v}_{1}, \ldots, \tilde{v}_{n}\right)$ satisfies $\tilde{v}_{i} \geq \bar{\rho}$ for $i \in\{1, \ldots, m(z)\}$. Consequently, for all $n \in N_{\Gamma^{(z)}}(\bar{\xi}) \cap S^{n-1}$,

$$
\langle n, \tilde{v}\rangle \leq-\bar{\rho}\left(\sum_{i=1}^{m(z)}\left|n_{i}\right|\right) \leq-\bar{\rho} .
$$

This shows that (3.7) holds true. In particular, due to Lemma 3.3, for every $z \in \Omega$ we get constants $r_{\Phi^{(z)}(z)}^{*}, T_{\Phi^{(z)}(z)}^{*}, K_{\Phi^{(z)}(z)}^{*}>0$ such that the conclusion of Lemma 3.3 holds for $\widetilde{F}^{(z)}$ on $\Gamma^{(z)}$.

Finally, note that in terms of the coordinates $\xi(t) \doteq \Phi^{(z)}(x(t))$, for $x(t) \in$ $B\left(z, \tilde{r}_{1}(z)\right)$, the differential inclusion (2.2) takes the form

$$
\dot{\xi}(t) \in \widetilde{F}^{(z)}(t, \xi(t))=D \Phi^{(z)}\left(\left(\Phi^{(z)}\right)^{-1}(\xi(t))\right) \cdot F\left(t,\left(\Phi^{(z)}\right)^{-1}(\xi(t))\right) .
$$

3. For every $z \in \Omega$, let $r_{z}>0$ be such that the following are satisfied

$$
B\left(z, r_{z}\right) \subseteq V_{z}, r_{z}<\tilde{r}_{1}(z), r_{z} \leq \frac{r_{\Phi^{(z)}(z)}^{*}}{\operatorname{Lip}\left(\Phi^{(z)}\right)}
$$


where $\tilde{r}_{1}(z)$ is the same as in part 1 . of this proof, and $r_{\Phi^{(z)}(z)}^{*}>0$ is given by Lemma 3.3. Consider the finite open subcovering of $\Omega$ given by

$$
\left\{B\left(z_{\ell}, r_{z_{\ell}}\right) \mid \ell=1, \ldots, N^{*}\right\} \doteq\left\{B_{\ell} \mid \ell=1, \ldots, N^{*}\right\} \supset \Omega .
$$

Let $2 \delta>0$ be the Lebesgue number of the above covering and $L_{\phi}$ denotes a common Lipschitz constant to all the diffeomorphisms $\Phi^{\ell}(\cdot) \doteq \Phi^{\left(z_{\ell}\right)}(\cdot)$ and their inverses, i.e.

$$
L_{\phi} \doteq \max _{\ell=1, \ldots, N^{*}}\left\{\operatorname{Lip}\left(\Phi^{\ell}\right)+\operatorname{Lip}\left(\left(\Phi^{\ell}\right)^{-1}\right)\right\} .
$$

Call $\widetilde{L}_{\ell}$ the Lipschitz constant of $\widetilde{F}_{\ell}(t, \cdot) \doteq \widetilde{F}^{\left(z_{\ell}\right)}(t, \cdot)$, and define

$$
\begin{aligned}
& \widetilde{L} \doteq \max _{\ell=1, \ldots, N^{*}} \widetilde{L}_{\ell}, \\
& \widetilde{M} \doteq L_{\phi} M \geq \max _{\ell=1, \ldots, N^{*}} \sup \left\{\widetilde{F}_{\ell}(t, \xi) \mid \xi \in \Phi^{\ell}\left(B\left(z_{\ell}, r_{z_{\ell}}\right)\right), t \in[0, T]\right\} .
\end{aligned}
$$

4. Let $\nu$ be a positive integer such that,

$$
\Delta t \doteq \frac{T}{\nu}<\min \left\{\frac{\delta}{2 M}, \min _{\ell=1, \ldots, N^{*}} T_{\Phi^{\left(z_{\ell}\right)}\left(z_{\ell}\right)}^{*}\right\},
$$

and let

$$
\widetilde{K} \doteq 2 \widetilde{M}+2 .
$$

Note that by Lemma $3.3 \nu$ and $\widetilde{K}$ depend only on the data. Let the $F$-trajectory $x^{*}(\cdot)$ be given. Then, choose a sequence of indices $j(i), i=1, \ldots, \nu$, such that

$$
B\left(x^{*}((i-1) \Delta t), \delta\right) \subset B\left(z_{j(i)}, r_{z_{j(i)}}\right) .
$$

This can be done by the Lebesgue covering Lemma. Finally, define $\gamma \doteq \sqrt[\nu]{\beta}$ and

$$
\varepsilon_{0} \doteq\left(\frac{\min \left\{\frac{\delta}{2}, \min _{i=1, \ldots, \nu} \varepsilon_{\Phi^{j(i)}\left(z_{j(i)}\right)}^{*}\right\}}{\left(L_{\phi}^{2} \widetilde{K}\right)^{\nu}}\right)^{\frac{1}{\beta}}
$$

where $\varepsilon_{\Phi^{j(i)}\left(z_{j(i)}\right)}^{*}$ is obtained from Lemma 3.3.

5 . Consider the first interval $[0, \Delta t]$. We see that

$$
x^{*}([0, \Delta t]) \subset B\left(x^{*}(0), \delta\right) \cap \Omega \subset \Omega \cap V_{j(1)} .
$$

Hence,

$$
\xi_{1}^{*}(t) \doteq \Phi^{j(1)}\left(x^{*}(t)\right) \in\left(\mathbb{R}_{+}^{m_{j(1)}} \times \mathbb{R}^{n-m_{j(1)}}\right), \quad \text { for all } t \in[0, \Delta t] .
$$

Also, since $\left|x_{0}-x^{*}(0)\right| \leq \varepsilon_{0} \leq \delta / 2$, we have that

$$
x_{0} \in V_{j(1)},
$$

and, since $\left|x_{0}-x^{*}(0)\right| \leq \varepsilon_{0} \leq \frac{\varepsilon_{\Phi^{j(1)}\left(z_{j(1)}\right)}^{*}}{L_{\phi}}$, we have that

$$
\left|\Phi^{j(1)}\left(x^{*}(0)\right)-\Phi^{j(1)}\left(x_{0}\right)\right| \leq \varepsilon_{\Phi^{j(1)}\left(z_{j(1)}\right)}^{*} .
$$

We consider two different cases. 
Case 1. $x_{0} \neq x^{*}(0)$.

Define $\varepsilon \doteq\left|x_{0}-x^{*}(0)\right|$. Then we can construct the $\widetilde{F}_{j(1)}$-trajectory $\xi_{1}$ by applying Lemma 3.3.(i)

$$
\xi_{1}:[0, \Delta t] \rightarrow \operatorname{Int}\left(\mathbb{R}_{+}^{m_{j(1)}} \times \mathbb{R}^{n-m_{j(1)}}\right),
$$

such that

$$
\left\|\xi_{1}(t)-\xi_{1}^{*}(t)\right\|_{\mathbf{L}^{\infty}([0, \Delta t])} \leq \widetilde{K}\left|\Phi^{j(1)}\left(x^{*}(0)\right)-\Phi^{j(1)}\left(x_{0}\right)\right|^{\gamma} .
$$

Finally, let $x_{1}(t):[0, \Delta t] \rightarrow \operatorname{Int} \Omega$ be given by $x_{1}(t) \doteq\left(\Phi_{j(1)}\right)^{-1}\left(\xi_{1}(t)\right)$.

From (3.29) we get

$$
\left\|x_{1}-x^{*}\right\|_{\mathbf{L}^{\infty}([0, \Delta t])} \leq L_{\phi}\left\|\xi_{1}(t)-\xi_{1}^{*}(t)\right\|_{\mathbf{L}^{\infty}([0, \Delta t])} \leq L_{\phi} \widetilde{K}\left(L_{\phi} \varepsilon_{0}\right)^{\gamma} \leq \delta .
$$

Hence, by the choice of $j(2), x^{*}(\Delta t), x_{1}(\Delta t) \in B_{j(2)} \cap \Omega$.

Case 2. $x_{0}=x^{*}(0)$.

Then we fix any $\varepsilon<\varepsilon_{0}$, by applying Lemma 3.3.(ii) we can construct $x_{1}:[0, \Delta t] \rightarrow \operatorname{Int} \Omega$ such that

$$
\left\|x_{1}-x^{*}\right\|_{\mathbf{L}_{([0, \Delta t])}^{\infty}} \leq L_{\Phi}^{2} \widetilde{K} \varepsilon \leq \delta .
$$

6. We are now ready to construct $x(\cdot)$ on each subinterval $[(k-1) \Delta t, k \Delta t]$, for $k=1, \ldots, \nu$ by repeating the previous construction. This yields an $F$ - trajectory $x_{k}$ defined on the interval $[(k-1) \Delta t, k \Delta t]$, with the property that $x_{k}(t) \in \operatorname{Int} \Omega$, and

$\left\|x_{k}-x^{*}\right\|_{\mathbf{L}^{\infty}([(k-1) \Delta t, k \Delta t])} \leq \max \left\{L_{\phi}^{2} \widetilde{K}\left|x_{k}((k-1) \Delta t)-x^{*}((k-1) \Delta t)\right|^{\gamma}, L_{\phi}^{2} \widetilde{K} \varepsilon\right\}$.

Finally, define the $F$-trajectory,

$$
x(t) \doteq \sum_{i=1}^{\nu} x_{i}(t) \chi_{[(i-1) \Delta t, i \Delta t[}(t) .
$$

Clearly, we have that $x(t) \in \operatorname{Int} \Omega$. The $\mathbf{L}^{\infty}$ distance between $x$ and $x^{*}$ can be estimated as follows,

$$
\begin{aligned}
\left\|x^{*}-x\right\|_{\mathbf{L}^{\infty}([0, \nu \Delta t])} & \leq \widetilde{K} L_{\phi}^{2} \max \left\{\left|x^{*}((\nu-1) \Delta t)-x((\nu-1) \Delta t)\right|^{\gamma}, \varepsilon\right\} \\
& \leq\left(\widetilde{K} L_{\phi}^{2}\right)^{2} \max \left\{\left|x^{*}((\nu-2) \Delta t)-x((\nu-2) \Delta t)\right|^{\gamma^{2}}, \varepsilon^{\gamma}\right\} \\
& \leq\left(\widetilde{K} L_{\phi}^{2}\right)^{\nu} \varepsilon^{\gamma^{\nu}} .
\end{aligned}
$$

We get the estimate $(3.2)$ with $K=\left(\widetilde{K} L_{\phi}^{2}\right)^{\nu}$.

\section{Semicontinuous solutions to Hamilton-Jacobi-Bellman equations with state constraints}

Consider the following optimal control problem in the Bolza form:

$$
\text { Minimize } g(x(T))+\int_{0}^{T} L(t, x(t), u(t)) d t,
$$


over measurable maps $u:[0, T] \rightarrow \mathbb{R}^{m}$ and absolutely continuous solutions $x:[0, T] \rightarrow \mathbb{R}^{n}$ of the control system

$$
\left\{\begin{array}{l}
\dot{x}(t)=f(t, x(t), u(t)) \quad \text { a.e. in }[0, T] \\
x(0)=x_{0}
\end{array}\right.
$$

satisfying the constraints

$$
x(t) \in \Omega, \quad \text { for all } t \in[0, T] \quad \text { and } \quad u(t) \in U(t) \text { a.e. in }[0, T],
$$

where the maps $f:[0, T] \times \mathbb{R}^{n} \times \mathbb{R}^{m} \rightarrow \mathbb{R}^{n}, g: \mathbb{R}^{n} \rightarrow \mathbb{R} \cup\{+\infty\}, L:[0, T] \times$ $\mathbb{R}^{n} \times \mathbb{R}^{m} \rightarrow\left[0,+\infty\left[\right.\right.$, the set-valued map $U:[0, T] \rightsquigarrow \mathbb{R}^{m}$, the compact subset $\Omega \subset \mathbb{R}^{n}$ and the initial state $x_{0} \in \mathbb{R}^{n}$ are given.

We denote by $V:[0, T] \times \Omega \rightarrow \mathbb{R} \cup\{+\infty\}$ the value function of the problem $(C P)$, that is for all $(t, x) \in[0, T] \times \Omega, V(t, x)$ is the infimum of $g(y(T))+\int_{t}^{T} L(s, y(s), u(s)) d s$ over trajectory-control pairs $(y(\cdot), u(\cdot))$ of the control problem

$$
\left\{\begin{array}{l}
\dot{y}(s)=f(s, y(s), u(s)), \quad u(s) \in U(s), \quad \text { for a.e. } s \in[t, T], \quad\left(C P_{t, x}\right) \\
y(s) \in \Omega \text { for all } s \in[t, T] \quad \text { and } y(t)=x
\end{array}\right.
$$

It is well-known that the value function satisfies the Hamilton-Jacobi equation,

$$
\left\{\begin{array}{l}
-V_{t}(t, x)+H_{1}\left(t, x,-V_{x}(t, x)\right)=0 \\
V(T, x)=g(x) \text { for all } x \in \mathbb{R}^{n}
\end{array}\right.
$$

in a generalized sense, where $H_{q}:[0, T] \times \Omega \times \mathbb{R}^{n} \rightarrow \mathbb{R}$ is the Hamiltonian of the control problem $(C P)$ defined by

$$
H_{q}(t, x, p)=\sup _{u \in U(t)}\{\langle p, f(t, x, u)\rangle-q L(t, x, u)\} .
$$

Furthermore, in the case where $g(\cdot)$ incorporates endpoint constraints the value function may be discontinuous. It is the purpose of this section to show that, even if the value function is only lower semicontinuous, it is the unique solution of the Hamilton-Jacobi equation ( $H J E)$.

Lower semicontinuous solutions to Hamilton-Jacobi equations in the presence of state constraints were investigated by Frankowska and Plaskacz (see $[11,12])$ by using the subdifferential of the value function. However, if the data is only measurable in the time variable, then, like it was done in [13] in the absence of state constraints, one has to adapt the definition of solutions of $(H J E)$ in order to have uniqueness. Conditions under which our definition is equivalent to the definition involving only the subdifferentials are also discussed in [13].

Definition. A lower semicontinuous function $W:[0, T] \times \Omega \rightarrow \mathbb{R} \cup\{+\infty\}$ is a lower semicontinuous solution to $(H J E)$ if $W(T, \cdot)=g(\cdot)$ and there exists a 
set $D \subset[0, T]$ of full measure such that for all $t \in D$,

$$
\text { for all } x \in \partial \Omega, \text { for all } \begin{aligned}
\left(p_{t}, p_{x}, q\right) & \in\left[T_{\mathcal{E}_{p} W}(t, x, W(t, x))\right]^{-}, \\
& -p_{t}+H_{-q}\left(t, x,-p_{x}\right) \geq 0,
\end{aligned}
$$

for all $x \in \operatorname{Int} \Omega$, for all $\left(p_{t}, p_{x}, q\right) \in\left[T_{\mathcal{E}_{p} W}(t, x, W(t, x))\right]^{-}$,

$$
-p_{t}+H_{-q}\left(t, x,-p_{x}\right)=0 .
$$

Remark 4.1. Note that by Proposition 2.1, if $W$ is a lower semicontinuous solution of the Hamilton-Jacobi equation, then in particular for all $t \in D$ and

for all $x \in \partial \Omega$, for all $\left(p_{t}, p_{x}\right) \in \partial_{-} W(t, x),-p_{t}+H_{1}\left(t, x,-p_{x}\right) \geq 0$, for all $x \in \operatorname{Int} \Omega$, for all $\left(p_{t}, p_{x}\right) \in \partial_{-} W(t, x),-p_{t}+H_{1}\left(t, x,-p_{x}\right)=0$.

We make the following assumptions on the data.

(H1) The set-valued map $U(\cdot)$ is measurable with closed, nonempty images;

(H2) There exists a constant $l>0$ such that, for all $t \in[0, T], u \in U(t)$, the maps $x \mapsto f(t, x, u)$ and $x \mapsto L(t, x, u)$ are locally $l$-Lipschitz continuous;

(H3) For all $x \in \mathbb{R}^{n}, u \in \mathbb{R}^{m}$, the maps $t \mapsto f(t, x, u)$ and $t \mapsto L(t, x, u)$ are Lebesgue measurable;

(H4) For all $t \in[0, T], x \in \Omega$, the maps $u \mapsto f(t, x, u)$ and $u \mapsto L(t, x, u)$ are continuous;

(H5) There exists $M>0$ such that, for all $(t, x) \in[0, T] \times \Omega$ and all $u \in$ $U(t),|f(t, x, u)|+|L(t, x, u)| \leq M$. Furthermore, for all $(t, x) \in[0, T] \times \Omega$, the set $\{(f(t, x, u), L(t, x, u)+r) \mid u \in U(t), r \geq 0\}$ is closed and convex;

(H6) $g: \Omega \rightarrow \mathbb{R} \cup\{+\infty\}$ is a lower semicontinuous function such that $\operatorname{Dom}(g) \neq \emptyset$. Moreover, for every $y \in \operatorname{Dom}(g)$, there exists a sequence $y_{k} \rightarrow y$ such that $y_{k} \in$ Int $\Omega$ for all $k$ and $\lim _{k \rightarrow \infty} g\left(y_{k}\right)=g(y)$;

(H7) For all $x \in \partial \Omega$, there exists $\rho>0$ such that for all $t \in[0, T]$,

$$
\sup _{u \in U(t)} \inf _{n \in N_{\Omega}(x) \cap S^{n-1}}\langle n, f(t, x, u)\rangle \geq \rho .
$$

Remark 4.2. Recall that the Bolza problem under state and enpoint constraints contains an additional restriction $x(T) \in \bar{C}$ for an open subset $C \subset \Omega$. If $g: \mathbb{R}^{n} \rightarrow \mathbb{R}$ is continuous, then setting

$$
\tilde{g}(x)=\left\{\begin{array}{cl}
g(x) & \text { if } x \in \bar{C} \\
+\infty & \text { otherwise }
\end{array}\right.
$$

we have the Bolza problem with lower semicontinuous function $\tilde{g}$ instead of $g$ satisfying (H6).

Before we state the main result of this section, let us introduce the following notations. We define the set-valued maps $F^{+}, F^{-}:[0, T] \times \mathbb{R}^{n} \times \mathbb{R} \rightsquigarrow$ $\mathbb{R}^{n} \times \mathbb{R}$ by

$$
\begin{gathered}
F^{+}(t, x, w) \doteq\{(f(t, x, u), L(t, x, u)+r) \mid u \in U(t), r \in[0, M-L(t, x, u)]\} \\
F^{-}(t, x, w) \doteq\{(f(t, x, u),-L(t, x, u)-r) \mid u \in U(t), r \in[0, M-L(t, x, u)]\} .
\end{gathered}
$$


Note that by the above assumptions, the set-valued maps $F^{+}$and $F^{-}$are $l$ Lipschitz in the $x$ variable, constant in $w^{1}$, measurable in $t$ and have compact convex images. Moreover, by assumptions (H2) and (H5) they also have the linear growth property with respect to $x$. Further we define,

$$
\begin{gathered}
A\left(t_{0}, x_{0}\right) \doteq\left\{u:\left[t_{0}, T\right] \rightarrow \mathbb{R}^{m} \text { measurable } \mid u(t) \in U(t)\right. \text { a.e. } \\
\text { and } \left.x\left(t ; t_{0}, x_{0}, u\right) \in \Omega \text { for all } t\right\},
\end{gathered}
$$

where we denote by $x(\cdot ; t, x, u)$ the unique solution of the control system $\left(C P_{t, x}\right)$ corresponding to the control $u(\cdot)$. We denote by $\mathcal{S}\left(t_{0}, z_{0}\right)$ the set of solutions of the differential inclusion

$$
\left\{\begin{array}{l}
\dot{z}(t) \in F^{+}(t, z(t)) \quad \text { a.e. in }\left[t_{0}, T\right] \\
z\left(t_{0}\right)=z_{0} .
\end{array}\right.
$$

Moreover, $\mathcal{S}_{\Omega}\left(t_{0}, z_{0}\right) \doteq\left\{z \in \mathcal{S}\left(t_{0}, z_{0}\right) \mid z(t) \in \Omega \times \mathbb{R}\right.$, for all $\left.t \in\left[t_{0}, T\right]\right\}$. It follows directly from a classical theorem due to Filippov (see for instance $[1$, Thm. 8.2.10]) that $z \in \mathcal{S}_{\Omega}\left(t_{0},\left(x_{0}, w_{0}\right)\right)$ if and only if there exist $u \in A\left(t_{0}, x_{0}\right)$ and a measurable $\eta:\left[t_{0}, T\right] \rightarrow\left[0,+\infty\left[\right.\right.$ such that $L\left(t, x\left(t ; t_{0}, x_{0}, u\right), u(t)\right)+\eta(t) \leq M$ for all $t$ and

$$
z(t)=\left(x\left(t ; t_{0}, x_{0}, u\right), w_{0}+\int_{t_{0}}^{t}\left(L\left(s, x\left(s ; t_{0}, x_{0}, u\right), u(s)\right)+\eta(s)\right) d s\right) .
$$

Furthermore, it is well-known that for all $\left(t_{0}, x_{0}\right) \in[0, T] \times \Omega$, the sets $\mathcal{S}\left(t_{0},\left(x_{0}, 0\right)\right)$ and $\mathcal{S}_{\Omega}\left(t_{0},\left(x_{0}, 0\right)\right)$ are compact subsets of $\mathcal{C}\left(\left[t_{0}, T\right] ; \mathbb{R}^{n+1}\right)$. Consequently, since the map $\varphi: \mathcal{S}_{\Omega}\left(t_{0},\left(x_{0}, 0\right)\right) \rightarrow \mathbb{R} \cup\{+\infty\}$, defined by

$$
\varphi(z)=g(x(T))+w(T), \quad \text { where } z(t)=(x(t), w(t)),
$$

is lower semicontinuous, it attains a minimum on the compact set $\mathcal{S}_{\Omega}\left(t_{0}\right.$, $\left.\left(x_{0}, 0\right)\right)$. Thus, again by Filippov's theorem ([1, Thm. 8.2.10]), there exists $\bar{u} \in A\left(t_{0}, x_{0}\right)$ such that

$$
V\left(t_{0}, x_{0}\right)=g\left(x\left(T ; t_{0}, x_{0}, \bar{u}\right)\right)+\int_{t_{0}}^{T} L\left(s, x\left(s ; t_{0}, x_{0}, \bar{u}\right), \bar{u}(s)\right) d s .
$$

Finally, let $W:[0, T] \times \Omega \rightarrow \mathbb{R} \cup\{+\infty\}$ be a function. The following set-valued map related with the epigraph of $W$ will be important in what follows. For $t \in[0, T]$, we define

$$
\mathcal{V}_{W}(t) \doteq\{(x, w) \mid x \in \Omega, w \geq W(t, x)\} .
$$

Using the above assumptions and these notations we will prove that the value function is the unique lower semicontinuous solution of the HamiltonJacobi equation. More precisely we have the following result:

Theorem 4.1. Let $\Omega \subset \mathbb{R}^{n}$ be such that (SC) is satisfied. Further assume that (H1)-(H7) hold true. Then for a lower semicontinuous function $W:[0, T] \times$ $\Omega \rightarrow \mathbb{R} \cup\{+\infty\}$ the following statements are equivalent:

(a) $W=V$;

${ }^{1}$ Later we use $F^{ \pm}$as the right hand side of a differential inclusion [see for instance (4.6)] therefore the constant dependence on $w$ is used. Compare with [11]. 
(b) $\mathcal{V}_{W}(\cdot)$ is absolutely continuous and $W$ is a lower semicontinuous solution of the Hamilton-Jacobi equation.

Proof. The proof of Theorem 4.1 is obtained from the Propositions below in the following manner.

Assume (a). Then Propositions $4.2-4.5$ prove that $(\mathrm{a}) \Longrightarrow(\mathrm{b})$. On the other hand assume (b). Then we obtain from the Propositions 4.6 and 4.7 that $(\mathrm{b}) \Longrightarrow(\mathrm{a})$.

As already mentioned, the fact that the value function is a lower semicontinuous solution of the Hamilton-Jacobi equation is known. Even though our assumptions differ slightly from the assumptions in the literature, we give a proof of this fact mainly for the sake of completeness. In the following we always assume that (H1)-(H7) are satisfied.

Proposition 4.2. The value function $V$ is lower semicontinuous.

Proof. Consider $\left(t_{0}, y_{0}\right) \in[0, T] \times \Omega$ and a sequence $\left\{\left(t_{n}, y_{n}\right)\right\}_{n \in \mathbb{N}}$ such that $\left(t_{n}, y_{n}\right) \rightarrow\left(t_{0}, y_{0}\right)$. If $\liminf _{n \rightarrow \infty} V\left(t_{n}, y_{n}\right)=+\infty$ then we are done. Therfore we assume that the above liminf is finite. Without loss of generality assume that $\lim _{n \rightarrow \infty} V\left(t_{n}, y_{n}\right)$ does exist. Then let $u_{n} \in A\left(t_{n}, y_{n}\right)$ be such that $g\left(x_{n}(T)\right)+\int_{t_{n}}^{T} L\left(s, x_{n}(s), u_{n}(s)\right) d s=V\left(t_{n}, y_{n}\right)$, where $x_{n}(\cdot) \doteq x\left(\cdot ; t_{n}, y_{n}, u_{n}\right)$. If necessary, we extend (or restrict, if $t_{n}<t_{0}$ ) $u_{n}(\cdot)$ to the interval $\left[t_{0}, T\right]$ by taking an arbitrary measurable selection of $U(\cdot)$ defined on the interval $\left[t_{0}, t_{n}[\right.$. Then we set,

$$
\begin{gathered}
z_{n}(t) \doteq\left(x_{n}(t), \int_{t_{0}}^{t} L\left(s, x_{n}(s), u_{n}(s)\right) d s\right) \\
\bar{z}_{n}(t) \doteq\left(x\left(t ; t_{0}, y_{0}, u_{n}\right), \int_{t_{0}}^{t} L\left(s, x\left(s ; t_{0}, y_{0}, u_{n}\right), u_{n}(s)\right) d s\right) .
\end{gathered}
$$

Using Grönwall's lemma, it is easy to prove that there exists $K>0$, such that

$$
\left\|z_{n}-\bar{z}_{n}\right\|_{\mathbf{L} \infty} \leq K\left(\left|t_{n}-t_{0}\right|+\left|y_{n}-y_{0}\right|\right) .
$$

Obviously, the sequence $\left\{\bar{z}_{n}\right\}_{n \in \mathbb{N}}$ is such that $\bar{z}_{n} \in \mathcal{S}\left(t_{0},\left(y_{0}, 0\right)\right)$. Since the set $\mathcal{S}\left(t_{0},\left(y_{0}, 0\right)\right)$ is compact, there exists a subsequence $\bar{z}_{n_{k}}$ converging uniformly to $\bar{z} \in \mathcal{S}\left(t_{0},\left(y_{0}, 0\right)\right)$. Then, by $(4.7)$, it is clear that $z_{n_{k}} \rightarrow \bar{z}$ uniformly. Since $\Omega$ is closed, it follows that $\bar{z} \in \mathcal{S}_{\Omega}\left(t_{0},\left(y_{0}, 0\right)\right)$. We deduce the inequalities

$$
V\left(t_{0}, y_{0}\right) \leq \varphi(\bar{z}) \leq \liminf _{k \rightarrow \infty} \varphi\left(z_{n_{k}}\right)=\lim _{k \rightarrow \infty} V\left(t_{n_{k}}, y_{n_{k}}\right)=\liminf _{n \rightarrow \infty} V\left(t_{n}, y_{n}\right),
$$

which completes the proof of the lower semicontinuity.

Having established the required regularity, we are going to use the basic properties of the value function, in order to show (4.3) and (4.4).

Proposition 4.3. There exists a subset $D \subset[0, T]$ of full measure such that for all $(t, x) \in D \times \Omega$ and for all $\left(p_{t}, p_{x}, q\right) \in\left[T_{\mathcal{E} p V}(t, x, V(t, x))\right]^{-}$,

$$
-p_{t}+H_{-q}\left(t, x,-p_{x}\right) \geq 0 \text {. }
$$


Proof. For this proof it is easier to consider the Mayer problem, i.e. we define the value function $V_{M}:[0, T] \times \Omega \rightarrow \mathbb{R} \cup\{+\infty\}$ by

$$
V_{M}\left(t_{0}, z_{0}\right) \doteq \inf \left\{g(x(T))+w(T) \mid(x, w) \doteq z \in \mathcal{S}_{\Omega}\left(t_{0}, z_{0}\right)\right\} .
$$

It is well-known that with this definition $V_{M}\left(t_{0},\left(x_{0}, 0\right)\right)=V\left(t_{0}, x_{0}\right)$ for all $\left(t_{0}, x_{0}\right) \in[0, T] \times \Omega$. Further, the reachable set of the control system (4.6) at time $t \in[0, T]$ is given by

$$
R\left(t, t_{0}\right)\left(z_{0}\right) \doteq\left\{z(t) \mid z \in \mathcal{S}\left(t_{0}, z_{0}\right)\right\} .
$$

Then, by [13, Thm. 2.5] there exists a set of full measure $\widetilde{D} \subset[0, T]$ such that for all $\left(t_{0}, x_{0}, w_{0}\right) \in \widetilde{D} \times \Omega \times \mathbb{R}_{+}$,

$$
\operatorname{Lim}_{h \rightarrow 0} \frac{R\left(t_{0}+h, t_{0}\right)\left(x_{0}, w_{0}\right)-\left(x_{0}, w_{0}\right)}{h}=F^{+}\left(t_{0},\left(x_{0}, w_{0}\right)\right) .
$$

Fix $\left(t_{0}, x_{0}\right) \in(\widetilde{D} \cap[0, T[) \times \Omega$. We first show that

$$
\inf _{\substack{u \in U\left(t_{0}\right) \\ r \in\left[0, M-L\left(t_{0}, x_{0}, u\right)\right]}} D_{\uparrow} V_{M}\left(t_{0},\left(x_{0}, 0\right)\right)\left(1, f\left(t_{0}, x_{0}, u\right), L\left(t_{0}, x_{0}, u\right)+r\right) \leq 0 .
$$

In order to do this, let $\bar{u} \in A\left(t_{0}, x_{0}\right)$ be such that

$$
V\left(t_{0}, x_{0}\right)=g(\bar{x}(T))+\bar{w}(T),
$$

where $\bar{x}(\cdot) \doteq x\left(\cdot ; t_{0}, x_{0}, \bar{u}\right)$ and $\bar{w}(t) \doteq \int_{t_{0}}^{t} L(s, \bar{x}(s), \bar{u}(s)) d s$. Further, we set $\bar{z} \doteq(\bar{x}, \bar{w})$ and $z_{0} \doteq\left(x_{0}, 0\right)$. Then it follows that for all $t \in\left[t_{0}, T\right]$,

$$
V_{M}(t, \bar{z}(t))-V_{M}\left(t_{0}, z_{0}\right)=0 .
$$

For $h_{n} \rightarrow 0+$, we define $v_{n} \doteq\left(\bar{z}\left(t_{0}+h_{n}\right)-\bar{z}\left(t_{0}\right)\right) / h_{n}$. Since $v_{n}$ is bounded, there exist subsequences, still denoted by $h_{n}$ and $v_{n}$, such that $v \doteq \lim _{n \rightarrow \infty} v_{n}$ exists. Hence, by (4.10) there exist $u_{0} \in U\left(t_{0}\right)$ and $r_{0} \in\left[0, M-L\left(t_{0}, x_{0}, u_{0}\right)\right]$ such that $v=\left(f\left(t_{0}, x_{0}, u_{0}\right), L\left(t_{0}, x_{0}, u_{0}\right)+r_{0}\right)$. Using (4.12) we find that

$$
\begin{aligned}
& D_{\uparrow} V_{M}\left(t_{0}, z_{0}\right)\left(1, f\left(t_{0}, x_{0}, u_{0}\right), L\left(t_{0}, x_{0}, u_{0}\right)+r_{0}\right) \\
& \quad \leq \lim _{n \rightarrow+\infty} \frac{V_{M}\left(t_{0}+h_{n}, \bar{z}\left(t_{0}+h_{n}\right)\right)-V_{M}\left(t_{0}, z_{0}\right)}{h_{n}}=0 .
\end{aligned}
$$

Having established (4.11), it follows from the identity (2.1) that

$$
\left(1, f\left(t_{0}, x_{0}, u_{0}\right), L\left(t_{0}, x_{0}, u_{0}\right)+r_{0}, 0\right) \in T_{\mathcal{E}_{p} V_{M}}\left(t_{0}, z_{0}, V_{M}\left(t_{0}, z_{0}\right)\right) .
$$

Furthermore it is not difficult to verify that this implies that

$$
\left(1, f\left(t_{0}, x_{0}, u_{0}\right),-L\left(t_{0}, x_{0}, u_{0}\right)-r_{0}\right) \in T_{\mathcal{E} p V}\left(t_{0}, x_{0}, V\left(t_{0}, x_{0}\right)\right)
$$

and therefore for all $\left(p_{t}, p_{x}, q\right) \in\left[T_{\mathcal{E} p V}\left(t_{0}, x_{0}, V\left(t_{0}, x_{0}\right)\right)\right]^{-}$,

$$
p_{t}+\left\langle p_{x}, f\left(t_{0}, x_{0}, u_{0}\right)\right\rangle-q\left(L\left(t_{0}, x_{0}, u_{0}\right)+r_{0}\right) \leq 0 .
$$

Further, since $\left(0,0, \mathbb{R}_{+}\right) \subset T_{\mathcal{E} p V}\left(t_{0}, x_{0}, V\left(t_{0}, x_{0}\right)\right)$, therefore $q \leq 0$, and consequently,

$$
p_{t}+\left\langle p_{x}, f\left(t_{0}, x_{0}, u_{0}\right)\right\rangle-q L\left(t_{0}, x_{0}, u_{0}\right) \leq 0,
$$

which allows to conclude that 


$$
-p_{t}+H_{-q}\left(t_{0}, x_{0},-p_{x}\right) \geq 0 \text {. }
$$

Proposition 4.4. There exists a subset $D \subset[0, T]$ of full measure, such that for all $(t, x) \in D \times \operatorname{Int} \Omega$ and for all $\left(p_{t}, p_{x}, q\right) \in\left[T_{\mathcal{E} p V}(t, x, V(t, x))\right]^{-}$,

$$
-p_{t}+H_{-q}\left(t, x,-p_{x}\right) \leq 0 \text {. }
$$

Proof. $V_{M}$ is defined by (4.8), the reachable set is given by (4.9) and the set $\widetilde{D}$ is defined as in the proof of Proposition 4.3. We fix $\left.\left.\left(t_{0}, x_{0}\right) \in(\widetilde{D} \cap] 0, T\right]\right) \times$ Int $\Omega$. First note that since $x_{0} \in$ Int $\Omega$, there exists $\bar{h}>0$ such that for all $h \in[0, \bar{h}]$ and all $w_{0} \in \mathbb{R}_{+}, R\left(t_{0}-h, t_{0}\right)\left(x_{0}, w_{0}\right) \subset \Omega \times \mathbb{R}_{+}$.

Further, by (4.10) for all $u_{0} \in U\left(t_{0}\right), h_{n} \rightarrow 0+$, there exists a sequence $v_{n}$ converging to $v \doteq\left(-f\left(t_{0}, x_{0}, u_{0}\right),-L\left(t_{0}, x_{0}, u_{0}\right)\right)$ and satisfying for $z_{0} \doteq$ $\left(x_{0}, 0\right)$,

$$
z_{0}+h_{n} v_{n} \in R\left(t_{0}-h_{n}, t_{0}\right)\left(z_{0}\right) \text { for all } n .
$$

Without loss of generality we can assume that $h_{n} \leq \bar{h}$ for all $n$. Then it follows from the properties of the value function of the Mayer problem that,

$$
V_{M}\left(t_{0}-h_{n}, z_{0}+h_{n} v_{n}\right)-V_{M}\left(t_{0}, z_{0}\right) \leq 0 .
$$

We claim that, $D_{\uparrow} V_{M}\left(t_{0}, z_{0}\right)\left(-1,-f\left(t, x_{0}, u_{0}\right),-L\left(t_{0}, x_{0}, u_{0}\right)\right) \leq 0$. Indeed, this is a consequence of (4.13) since we have,

$$
D_{\uparrow} V_{M}\left(t_{0}, z_{0}\right)(-1, v) \leq \liminf _{n \rightarrow \infty} \frac{V_{M}\left(t_{0}-h_{n}, z_{0}+h_{n} v_{n}\right)-V_{M}\left(t_{0}, z_{0}\right)}{h_{n}} \leq 0 .
$$

Then, as $u_{0}$ was chosen arbitrarily, it follows from the identity (2.1) that, for all $u_{0} \in U\left(t_{0}\right),\left(-1,-f\left(t_{0}, x_{0}, u_{0}\right),-L\left(t_{0}, x_{0}, u_{0}\right), 0\right) \in T_{\mathcal{E} p V_{M}}\left(t_{0}, z_{0}, V_{M}\left(t_{0}, z_{0}\right)\right)$. This implies that $\left(-1,-f\left(t_{0}, x_{0}, u_{0}\right), L\left(t_{0}, x_{0}, u_{0}\right)\right) \in T_{\mathcal{E} p V}\left(t_{0}, x_{0}, V\left(t_{0}, x_{0}\right)\right)$ and consequently, for all $\left(p_{t}, p_{x}, q\right) \in\left[T_{\mathcal{E} p V}\left(t_{0}, x_{0}, V\left(t_{0}, x_{0}\right)\right)\right]^{-}$,

$$
-p_{t}+H_{-q}\left(t_{0}, x_{0},-p_{x}\right) \leq 0 \text {. }
$$

Proposition 4.5. $\mathcal{V}_{V}$ is an absolutely continuous set-valued map.

Proof. Consider $t_{0}, \tau \in[0, T]$ such that $t_{0}<\tau$. Let $\left(x_{0}, v_{0}\right) \in \mathcal{V}_{V}\left(t_{0}\right)$ and $\bar{u} \in A\left(t_{0}, x_{0}\right)$ be such that $g(\bar{x}(T))+\int_{t_{0}}^{T} L(s, \bar{x}(s), \bar{u}(s)) d s=V\left(t_{0}, x_{0}\right)$, where $\bar{x}(\cdot) \doteq x\left(\cdot ; t_{0}, x_{0}, \bar{u}\right)$. Then it follows from the properties of the value function that

$$
V(\tau, \bar{x}(\tau))=V\left(t_{0}, x_{0}\right)-\int_{t_{0}}^{\tau} L(s, \bar{x}(s), \bar{u}(s)) d s \leq V\left(t_{0}, x_{0}\right) .
$$

Hence, $\left(\bar{x}(\tau), v_{0}\right) \in \mathcal{V}_{V}(\tau)$. Consequently,

$$
\left(x_{0}, v_{0}\right) \in \mathcal{V}_{V}(\tau)+\left(\tau-t_{0}\right) M B .
$$


On the other hand, let $\left(x_{1}, v_{1}\right) \in \mathcal{V}_{V}(\tau)$. Using the outward pointing condition (4.5) and the viability theorem (Thm. 2.2), it follows that there exists a process $(x(\cdot), u(\cdot))$ solving the following viability problem:

$$
\left\{\begin{array}{l}
\dot{x}(t)=f(t, x(t), u(t)) \quad u(t) \in U(t) \quad \text { a.e. in }\left[t_{0}, \tau\right] \\
x(t) \in \Omega \quad \text { for all } t \in\left[t_{0}, \tau\right] \\
x(\tau)=x_{1} .
\end{array}\right.
$$

Then it follows from the properties of the value function that

$$
V\left(t_{0}, x\left(t_{0}\right)\right) \leq V\left(\tau, x_{1}\right)+\int_{t_{0}}^{\tau} L(s, x(s), u(s)) d s .
$$

Thus $\left(x\left(t_{0}\right), v_{1}+\int_{t_{0}}^{\tau} L(s, x(s), u(s)) d s\right) \in \mathcal{V}_{V}\left(t_{0}\right)$ and we deduce that

$$
\left(x_{1}, v_{1}\right) \in \mathcal{V}_{V}\left(t_{0}\right)+\left(\tau-t_{0}\right) M B \text {. }
$$

The absolute continuity of $\mathcal{V}_{V}(\cdot)$ follows immediately from (4.14) and (4.15).

It is clear that the Propositions 4.2-4.5 imply that in Theorem 4.1 (a) $\Longrightarrow(\mathrm{b})$. In the remainder of this section, we are going to prove the opposite implication. This will be achieved by proving the following two comparison results.

Proposition 4.6. Let $W:[0, T] \times \Omega \rightarrow \mathbb{R} \cup\{+\infty\}$ be a lower semicontinuous solution of the Hamilton-Jacobi equation such that $\mathcal{V}_{W}(\cdot)$ is absolutely continuous. Then for all $(t, x) \in[0, T] \times \Omega, V(t, x) \leq W(t, x)$.

Proof. We do the proof in two steps. First we show that the viability theorem (Thm. 2.2) can be applied to $F^{-}$, then we deduce the comparison result from the viability theorem.

1. We show that for almost every $t_{0} \in[0, T], x_{0} \in \Omega$ and $w_{0} \geq W\left(t_{0}, x_{0}\right)$,

$$
\left(\{1\} \times F^{-}\left(t_{0}, x_{0}\right)\right) \cap \overline{c o} T_{\mathrm{Gr}} \mathcal{V}_{W}\left(t_{0}, x_{0}, w_{0}\right) \neq \emptyset .
$$

Let $t_{0} \in D$, where $D \subset[0, T]$ is the subset of full measure from the definition of a lower semicontinuous solution, $x_{0} \in \Omega$ and $w_{0} \geq W\left(t_{0}, x_{0}\right)$. First, note that $\operatorname{Gr} \mathcal{V}_{W}=\mathcal{E} p W$ and that, for all $w_{0} \geq W\left(t_{0}, x_{0}\right)$,

$$
T_{\mathcal{E} p W}\left(t_{0}, x_{0}, W\left(t_{0}, x_{0}\right)\right) \subset T_{\mathcal{E} p W}\left(t_{0}, x_{0}, w_{0}\right) .
$$

Hence it suffices to show (4.16) for $w_{0}=W\left(t_{0}, x_{0}\right)$. Assume on the contrary that (4.16) does not hold. Then, by the separation theorem, there exists $\left(p_{t}, p_{x}, q\right) \in\left[T_{\mathcal{E} p W}\left(t_{0}, x_{0}, W\left(t_{0}, x_{0}\right)\right)\right]^{-}$such that

$$
\inf _{\substack{u \in U\left(t_{0}\right) \\ r \in\left[0, M-L\left(t_{0}, x_{0}, u\right)\right]}}\left\{p_{t}+\left\langle p_{x}, f\left(t_{0}, x_{0}, u\right)\right\rangle-q\left(L\left(t_{0}, x_{0}, u\right)+r\right)\right\}>0 .
$$

Further note that $(0,0) \times \mathbb{R}_{+} \subset T_{\mathcal{E}_{p} W}\left(t_{0}, x_{0}, w_{0}\right)$ for all $\left(t_{0}, x_{0}, w_{0}\right) \in \mathcal{E} p W$ and therefore $q \leq 0$ and we get,

$$
-p_{t}+H_{-q}\left(t_{0}, x_{0},-p_{x}\right)<0,
$$

which contradicts the assumption that $W$ is a lower semicontinuous solution of the Hamilton-Jacobi equation. 
2. We show that for all $\left(t_{0}, x_{0}\right) \in[0, T] \times \Omega, V\left(t_{0}, x_{0}\right) \leq W\left(t_{0}, x_{0}\right)$.

For $t_{0}=T$ the statement is obvious. Let $\left(t_{0}, x_{0}\right) \in[0, T[\times \Omega$. Then by the first step and Theorem 2.2, we know that there exists $z(\cdot)$, solution of

$$
\begin{cases}\dot{z}(t) \in F^{-}(t, z(t)), & \text { a.e. in }\left[t_{0}, T\right], \\ z(t) \in \mathcal{V}_{W}(t), & \text { for all } t \in\left[t_{0}, T\right], \\ z\left(t_{0}\right)=\left(x_{0}, W\left(t_{0}, x_{0}\right)\right) . & \end{cases}
$$

Thus there exists $u \in A\left(t_{0}, x_{0}\right)$ and a positive integrable function $\eta(\cdot)$ such that for all $t \in\left[t_{0}, T\right]$,

$$
z(t)=\left(x(t), W\left(t_{0}, x_{0}\right)-\int_{t_{0}}^{t}(L(s, x(s), u(s))+\eta(s)) d s\right),
$$

where $x(\cdot) \doteq x\left(\cdot ; t_{0}, x_{0}, u\right)$. Furthermore, for all $t \in\left[t_{0}, T\right]$,

$$
W\left(t_{0}, x_{0}\right)-\int_{t_{0}}^{t}(L(s, x(s), u(s))+\eta(s)) d s \geq W(t, x(t)),
$$

and thus in particular

$$
\begin{aligned}
W\left(t_{0}, x_{0}\right) & \geq W(T, x(T))+\int_{t_{0}}^{T} L(s, x(s), u(s)) d s \\
& =g(x(T))+\int_{t_{0}}^{T} L(s, x(s), u(s)) d s \geq V\left(t_{0}, x_{0}\right) .
\end{aligned}
$$

Proposition 4.7. Let $W:[0, T] \rightarrow \mathbb{R} \cup\{+\infty\}$ be a lower semicontinuous solution of the Hamilton-Jacobi equation such that $\mathcal{V}_{W}(\cdot)$ is absolutely continuous. Then for all $(t, x) \in[0, T] \times \Omega, W(t, x) \leq V(t, x)$.

Proof. The main ingredients of the proof are the neighboring feasible trajectories theorem (Thm. 3.1) and the viability theorem (Thm. 2.2).

Fix $\left(t_{0}, x_{0}\right) \in[0, T] \times \Omega$. Since the case $t_{0}=T$ is obvious, we assume from now on that $t_{0}<T$. Then let $u \in A\left(t_{0}, x_{0}\right)$ be arbitrary and set $x_{u}(\cdot) \doteq$ $x\left(\cdot ; t_{0}, x_{0}, u\right)$. If $g\left(x_{u}(T)\right)=+\infty$, then obviously

$$
W\left(t_{0}, x_{0}\right) \leq g\left(x_{u}(T)\right)+\int_{t_{0}}^{T} L\left(s, x_{u}(s), u(s)\right) d s .
$$

Otherwise let $w_{u}(\cdot)$ denote the solution of

$$
\left\{\begin{array}{l}
\dot{w}(t)=-L\left(t, x_{u}(t), u(t)\right) \quad \text { a.e. in }\left[t_{0}, T\right] \\
w(T)=g\left(x_{u}(T)\right)
\end{array}\right.
$$

Then it is sufficient to show that

$$
W\left(t_{0}, x_{0}\right) \leq w_{u}\left(t_{0}\right)
$$

By assumption (H6) we can choose a sequence $y_{k} \stackrel{\text { Int } \Omega}{\longrightarrow} x_{u}(T)$ such that

$$
\lim _{k \rightarrow \infty} W\left(T, y_{k}\right)=\lim _{k \rightarrow \infty} g\left(y_{k}\right)=g\left(x_{u}(T)\right) .
$$

1. We show that there exists a sequence of feasible trajectories $\left\{x_{k}(\cdot)\right\}_{k}$ such that $x_{k}(T)=y_{k}, x_{k}\left(\left[t_{0}, T\right]\right) \subset$ Int $\Omega$ and $x_{k} \rightarrow x_{u}$ uniformly on $\left[t_{0}, T\right]$. 
Similarly, we show that there exists a sequence of functions $\left\{w_{k}(\cdot)\right\}_{k}$ such that $w_{k}(T)=g\left(y_{k}\right)$ and $w_{k} \rightarrow w_{u}$ uniformly on $\left[t_{0}, T\right]$.

It is not restrictive to assume that for all $k,\left|g\left(y_{k}\right)-g\left(x_{u}(T)\right)\right| \leq 1$. Then we define the intervals

$$
\begin{aligned}
\mathcal{I} & \doteq\left[g\left(x_{u}(T)\right)-1, g\left(x_{u}(T)\right)+1+M(1+2 T)\right] \\
\mathcal{I}^{\prime} & \doteq\left[g\left(x_{u}(T)\right)-1, g\left(x_{u}(T)\right)+1+2 M T\right]
\end{aligned}
$$

and the set $\widetilde{\Omega} \doteq \Omega \times \mathcal{I}$. It is obvious that $\widetilde{\Omega}$ satisfies (SC). Furthermore we define the set-valued maps $\widetilde{F}, \mathcal{F}:[0, T] \times \mathbb{R}^{n} \times \mathbb{R} \rightsquigarrow \mathbb{R}^{n+1}$, by $\widetilde{F}(t, x, w) \doteq$ $-F^{-}(T-t, x, w)$ and

$$
\begin{aligned}
& \mathcal{F}(t, x, w) \\
& \quad \doteq \begin{cases}\widetilde{F}(t, x, w), & \text { if } w \in \mathcal{I}^{\prime}, \\
\widetilde{F}(t, x, w)+\left\{(0, k) \mid k \in\left[-2\left(w-\left(g\left(x_{u}(T)\right)+1+2 M T\right), 0\right]\right\},\right. & \text { otherwise }\end{cases}
\end{aligned}
$$

It is a simple exercise to check that $\widetilde{\Omega}$ and $\mathcal{F}$ satisfy all the assumptions of Theorem 3.1. Further, it is obvious that $z_{u}:\left[0, T-t_{0}\right] \rightarrow \widetilde{\Omega}$ defined by

$$
z_{u}(t)=\left(x_{u}(T-t), w_{u}(T-t)\right)
$$

is an $\mathcal{F}$-trajectory. Then by Theorem 3.1, for arbitrary $\beta \in[0,1[$ there exist $K>0$ and $\varepsilon_{0}>0$ such that for all $k$ with $\left|\left(y_{k}, g\left(y_{k}\right)\right)-\left(x_{u}(T), w_{u}(T)\right)\right| \leq \varepsilon_{0}$ there exists an $\mathcal{F}$-trajectory $z_{k}:\left[0, T-t_{0}\right] \rightarrow$ Int $\widetilde{\Omega}$ such that $z_{k}(0)=\left(y_{k}, g\left(y_{k}\right)\right)$ and

$$
\left\|z_{k}-z_{u}\right\|_{\mathbf{L}^{\infty}\left(\left[0, T-t_{0}\right]\right)} \leq K\left|\left(y_{k}, g\left(y_{k}\right)\right)-\left(x_{u}(T), w_{u}(T)\right)\right|^{\beta} .
$$

Since $\left|\left(y_{k}, g\left(y_{k}\right)\right)-\left(x_{u}(T), w_{u}(T)\right)\right| \rightarrow 0$, this implies that $z_{k}$ converges uniformly to $z_{u}$. Note also that since $g\left(y_{k}\right) \in\left[g\left(x_{u}(T)\right)-1, g\left(x_{u}(T)\right)+1\right]$ and $\left|\dot{w}_{k}(t)\right| \leq 2 M, z_{k}$ is an $\widetilde{F}$-trajectory and there exists a measurable selection $u_{k}(\cdot)$ of $U(\cdot)$ and an integrable map $\eta_{k}:\left[0, T-t_{0}\right] \rightarrow \mathbb{R}$ such that, for $x_{k}(\cdot) \doteq x\left(\cdot ; T, y_{k}, u_{k}\right)$ and $w_{k}(\cdot)$ defined by

$$
w_{k}(t)=g\left(y_{k}\right)+\int_{t}^{T}\left(L\left(s, x_{k}(s), u_{k}(s)\right)+\eta_{k}(s)\right) d s,
$$

we have $z_{k}(t) \doteq\left(x_{k}(T-t), w_{k}(T-t)\right)$. We note in particular that

$$
\lim _{k \rightarrow \infty}\left(x_{k}\left(t_{0}\right), w_{k}\left(t_{0}\right)\right)=\left(x_{u}\left(t_{0}\right), w_{u}\left(t_{0}\right)\right) .
$$

2. We show that for all $k, W\left(t_{0}, x_{k}\left(t_{0}\right)\right) \leq w_{k}\left(t_{0}\right)$.

The claim will follow from Theorem 2.2. The difficulty is to show that this theorem can be applied.

We define $\widetilde{\mathcal{V}}_{W}(t) \doteq \mathcal{V}_{W}(T-t)$, which by Remark 2.1 is absolutely continuous. Then one shows in the same way as in the proof of Proposition 4.6 by using (4.4) and the separation theorem that there exists a subset $\widetilde{D} \subset[0, T]$ of full measure such that for all $(t, x) \in \widetilde{D} \times \operatorname{Int} \Omega$ and all $w \geq W(T-t, x)$,

$$
(\{1\} \times \widetilde{F}(t, x, w)) \subset \overline{c o} T_{\mathrm{Gr}} \widetilde{\mathcal{V}}_{W}(t, x, w) .
$$


Since we need an analogue of (4.19) also for $x \in \partial \Omega$, we consider the tube $\widehat{\mathcal{V}}_{W}(\cdot)$ defined by

$$
\widehat{\mathcal{V}}_{W}(t) \doteq \widetilde{\mathcal{V}}_{W}(t) \cup\left(\overline{\Omega^{c}} \times \mathbb{R}\right),
$$

which is also absolutely continuous. Then it is not difficult to verify that for all $(t, x, w) \in] 0, T] \times \overline{\Omega^{c}} \times \mathbb{R}$

$$
\left(\{1\} \times M \bar{B}^{n+1}\right) \cap T_{\mathrm{Gr}} \widehat{\mathcal{V}}_{W}(t, x, w) \neq \emptyset .
$$

Fix $k$ and define the set valued $\operatorname{map} F_{k}:[0, T] \times \mathbb{R}^{n} \times \mathbb{R} \rightarrow \mathbb{R}^{n+1}$ by

$$
\begin{aligned}
& F_{k}(t, x, w) \\
& \quad \doteq \begin{cases}\left(-f\left(T-t, x, u_{k}(T-t)\right), L\left(T-t, x, u_{k}(T-t)\right)+\eta_{k}(T-t)\right), & \text { if } x \in \operatorname{Int} \Omega, \\
M \bar{B}^{n+1} & \text { otherwise. }\end{cases}
\end{aligned}
$$

Then $F_{k}$ satisfies the assumptions of Theorem 2.2 and (4.19)-(4.20) imply that for almost all $t \in[0, T]$ and $(x, w) \in \widehat{\mathcal{V}}_{W}(t)$,

$$
\left(\{1\} \times F_{k}(t, x, w)\right) \cap \overline{c o} T_{\operatorname{Gr} \widehat{\mathcal{V}}_{W}}(t, x, w) \neq \emptyset .
$$

Furthermore, $z_{k}(\cdot)$ is the unique solution of the differential inclusion

$$
\left\{\begin{array}{l}
\dot{z}(t) \in F_{k}(t, z(t)) \quad \text { a.e. in }\left[0, T-t_{0}\right], \\
z(0)=\left(y_{k}, W\left(T, y_{k}\right)\right),
\end{array}\right.
$$

because $x_{k}(T-t) \in$ Int $\Omega$ for all $t \in\left[0, T-t_{0}\right]$ and $f$ and $L$ are Lipschitz with respect to $x$. Since $\left(y_{k}, W\left(T, y_{k}\right)\right) \in \widehat{\mathcal{V}}_{W}(0)$, by Theorem 2.2 , for all $t \in\left[0, T-t_{0}\right], z_{k}(t) \in \widehat{\mathcal{V}}_{W}(t)$. Further, using again $x_{k}(T-t) \in \operatorname{Int} \Omega$, we have actually that $z_{k}(t) \in \widetilde{\mathcal{V}}_{W}(t)$, which implies that for all $t \in\left[0, T-t_{0}\right], w_{k}(T-t) \geq$ $W\left(T-t, x_{k}(T-t)\right)$. Thus in particular for $t=T-t_{0}$,

$$
W\left(t_{0}, x_{k}\left(t_{0}\right)\right) \leq w_{k}\left(t_{0}\right) .
$$

3. We deduce from the lower semicontinuity of $W,(4.18)$ and (4.21) that

$$
W\left(t_{0}, x_{0}\right) \leq \liminf _{k \rightarrow \infty} W\left(t_{0}, x_{k}\left(t_{0}\right)\right) \leq \liminf _{k \rightarrow \infty} w_{k}\left(t_{0}\right)=w_{u}\left(t_{0}\right) .
$$

Hence we have shown (4.17), which ends the proof.

\section{Acknowledgments}

The authors are grateful to H. Frankowska and A. Bressan for providing them with lots of helpful advice and to R. B. Vinter for the hospitality and the encouragements during their stay at the Control and Power Group at Imperial College London in Spring 2011. The authors would also like to express their gratitude to anonymous referees whose comments significantly improved this paper. 


\section{References}

[1] Aubin, J.P., Frankowska, H.: Set-Valued Analysis, Systems and Control: Foundations and applications. Birkhäuser Boston, Inc., Boston (1990)

[2] Barron, E.N., Jensen, R.: Semicontinuous viscosity solutions for Hamilton-Jacobi equations with convex Hamiltonian. Commun. Partial Differ. Equ. 15, 1713$1742(1990)$

[3] Bettiol, P., Bressan, A., Vinter, R.: On trajectories satisfying a state constraint: $W^{1,1}$ estimates and counterexamples. SIAM J. Control Optim. 48, 46644679 (2010)

[4] Bettiol, P., Bressan, A., Vinter, R.: Estimates for trajectories confined to a cone in $\mathbb{R}^{n}$. SIAM J. Control Optim. 49, 21-41 (2011)

[5] Bettiol, P., Frankowska, H., Vinter, R.B.: $L^{\infty}$ estimates on trajectories confined to a closed subset. J. Diff. Equ. 252, 1912-1933 (2012)

[6] Bressan, A., Facchi, G.: Trajectories of differential inclusions with state constraints. J. Diff. Equ. 250, 2267-2281 (2011)

[7] Filippov, A.F.: Classical solutions of differential equations with multivalued right hand side. SIAM J. Control Optim. 5, 609-621 (1967)

[8] Frankowska, H.: Optimal trajectories associated with a solution of the contingent Hamilton-Jacobi equation. Appl. Math. Optim. 19, 291-311 (1989)

[9] Frankowska, H.: Lower semicontinuous solutions of Hamilton-Jacobi-Bellman equations. SIAM J. Control Optim. 31, 257-272 (1993)

[10] Frankowska, H., Plaskacz, S.: A measurable upper semicontinuous viability theorem for tubes. Nonlinear Anal. 26, 565-582 (1996)

[11] Frankowska, H., Plaskacz, S.: Semicontinuous solutions of Hamilton Jacobi equations with state constraints. In: Differential Inclusions and Optimal Control. Lecture Notes in Nonlinear Analysis, vol. 2, pp. 145-161. J. Schauder Center for Nonlinear Studies, Toruri (1998)

[12] Frankowska, H., Plaskacz, S.: Semicontinuous solutions of Hamilton-JacobiBellman equations with degenerate state constraints. J. Math. Anal. Appl. 251, 818-838 (2000)

[13] Frankowska, H., Plaskacz, S., Rzeżuchowski, T.: Measurable viability theorems and the Hamilton-Jacobi-Bellman equation. J. Diff. Equ. 116, 265-305 (1995)

[14] Frankowska, H., Rampazzo, F.: Filippov's and Filippov-Ważewski's theorems on closed domains. J. Diff. Equ. 161, 449-478 (2000)

[15] Frankowska, H., Vinter, R.B.: Existence of neighboring feasible trajectories: applications to dynamic programming for state constrained optimal control problems. J. Optim. Theory Appl. 104, 21-40 (2000) 
[16] Soner, H.M.: Optimal control problems with state-space constraints I. SIAM J. Control Optim. 24, 552-562 (1986)

[17] Soner, H.M.: Optimal control problems with state-space constraints II. SIAM J. Control Optim. 24, 1110-1122 (1986)

[18] Vinter, R.B.: Optimal Control. Birkhäuser, Boston (2000)

Giancarlo Facchi

Department of Mathematics

The Pennsylvania State University

University Park

PA 16802

USA

e-mail: facchi@math.psu.edu

Daniel Hoehener

Institut de Mathématiques de Jussieu

Université Pierre et Marie Curie (Paris 6)

Case 247, Combinatoire et Optimisation

4 place Jussieu

75252 Paris cedex 05

France

e-mail: hoehener@math.jussieu.fr

Received: 21 November 2011.

Accepted: 6 September 2012. 